\title{
Comparing cosmic shear measures
}

\section{Optimizing the information content of cosmic shear data vectors}

\author{
T. Eifler ${ }^{1}$, M. Kilbinger ${ }^{1,2}$, and P. Schneider ${ }^{1}$ \\ 1 Argelander-Institut für Astronomie, Universität Bonn, Auf dem Hügel 71, 53121 Bonn, Germany \\ e-mail: tim.eifler@astro.uni-bonn.de \\ 2 Institut d'Astrophysique de Paris, 98bis boulevard Arago, 75014 Paris, France
}

Received 29 August 2007 / Accepted 12 January 2008

\begin{abstract}
Aims. We introduce an optimized data vector of cosmic shear measures $(\mathcal{N})$. This data vector has high information content, is not sensitive to B-mode contamination, and only shows small correlation between data points of different angular scales.

Methods. We show that a data vector of the two-point correlation function (2PCF), hereafter denoted as $\boldsymbol{\xi}$, in general contains more information on cosmological parameters compared to a data vector of the aperture mass dispersion, hereafter referred to as $\left\langle\boldsymbol{M}_{\mathrm{ap}}^{2}\right\rangle$. The reason for this is the fact that $\left\langle\boldsymbol{M}_{\text {ap }}^{2}\right\rangle$ lacks the information of the convergence power spectrum $\left(\mathcal{P}_{K}\right)$ on large angular scales, which is contained in $\boldsymbol{\xi}$. Nevertheless, $\left\langle\boldsymbol{M}_{\text {ap }}^{2}\right\rangle$ has valuable properties (small correlation between data points of different angular scales, not sensitive to B-mode contamination) that one wants to preserve in an optimized data vector. Therefore we combine $\boldsymbol{\xi}$ and $\left\langle\boldsymbol{M}_{\mathrm{ap}}^{2}\right\rangle$ to a new data vector $\mathcal{N}=\left(\left\langle\boldsymbol{M}_{\text {ap }}^{2}\right\rangle\left(\theta_{1}\right), \ldots,\left\langle\boldsymbol{M}_{\text {ap }}^{2}\right\rangle\left(\theta_{n}\right), \xi_{+}\left(\theta_{0}\right)\right)$, which retains the advantages of $\left\langle\boldsymbol{M}_{\text {ap }}^{2}\right\rangle$ and is also sensitive to the large-scale information of $\mathcal{P}_{\kappa}$. We compare the information content of the three data vectors by performing a detailed likelihood analysis and use ray-tracing simulations to derive the covariance matrices. In the last part of the paper we contaminate all data vectors with B-modes on small angular scales and examine their robustness against this contamination.

Results. The combined data vector $\mathcal{N}$ strongly improves constraints on cosmological parameters compared to $\left\langle\boldsymbol{M}_{\mathrm{ap}}^{2}\right\rangle$. Although, the information content of $\xi$ is higher in the case of a pure E-mode signal, in the more realistic case where B-modes are present the $2 \mathrm{PCF}$ data vector is strongly contaminated and yields biased cosmological parameter estimates. The new data vector $\mathcal{N}$ shows to be robust against this contamination. Furthermore the individual data points of $\mathcal{N}$ show a much smaller correlation compared to $\boldsymbol{\xi}$, leading to an almost diagonal covariance matrix.
\end{abstract}

Key words. cosmology: theory - gravitational lensing - large-scale structure of Universe - methods: statistical

\section{Introduction}

Weak gravitational lensing by the large-scale structure (LSS) called cosmic shear has become a valuable tool in cosmology. Ever since the first detection of cosmic shear in 2000 (Bacon et al. 2000; Kaiser et al. 2000; van Waerbeke et al. 2000; Wittman et al. 2000), several surveys have been carried out with various depths and widths. The latest results show the ability of cosmic shear to constrain cosmological parameters, in particular $\sigma_{8}$ (e.g. van Waerbeke et al. 2005; Semboloni et al. 2006; Hoekstra et al. 2006; Schrabback et al. 2007; Hetterscheidt et al. 2007; Massey et al. 2007). In the near future these constraints will improve even more when upcoming surveys like Pan-STARRS or KIDS enable us to estimate the shear signal with less than $1 \%$ statistical error. This improvement in measuring cosmic shear should go along with an optimization of the data analysis. It is desirable to extract as much information as possible from the observational data and to derive constraints free of any contamination. Currently, most cosmic shear surveys only consider second-order shear statistics, for which all information is contained in the power spectrum of the convergence $\left(\mathcal{P}_{K}\right)$. Although $\mathcal{P}_{K}$ is not directly measureable, it is linearly related to second-order cosmic shear measures (e.g. the two-point correlation function and the aperture mass dispersion), which can be estimated from the distorted ellipticities of the observed galaxies. More precisely, all second-order measures are filtered versions of $\mathcal{P}_{K}$, and the corresponding filter functions determine how the information content of $\mathcal{P}_{K}$ is sampled.

It is the intention of this paper to compare several data vectors of cosmic shear measures and to create an optimal data vector with high information content, largely uncorrelated data points and only little sensitivity to a possible B-mode contamination. We first compare the information content of the twopoint correlation function (2PCF) and aperture mass dispersion $\left(\left\langle M_{\mathrm{ap}}^{2}\right\rangle\right)$. We prove a general statement that a data vector consisting of $2 \mathrm{PCF}$ data points $(\boldsymbol{\xi})$ always gives tighter constraints on cosmological models compared to a data vector consisting of $\left\langle M_{\mathrm{ap}}^{2}\right\rangle$ data points $\left(\left\langle\boldsymbol{M}_{\mathrm{ap}}^{2}\right\rangle\right)$ and we confirm this by a likelihood analysis of ray-tracing simulations. This result cannot come as a surprise since the $2 \mathrm{PCF}$ integrates over all scales of $\mathcal{P}_{K}$ and especially collects information on large angular scales, scales that are not taken into account by the aperture mass dispersion. Nevertheless $\left\langle M_{\text {ap }}^{2}\right\rangle$ has important advantages. First, it can be used to separate E-modes and B-modes (Crittenden et al. 2002; Schneider et al. 2002b), more precisely $\left\langle M_{\mathrm{ap}}^{2}\right\rangle$ is only sensitive to E-modes. Second, thanks to its narrow filter function, it provides highly localized information on $\mathcal{P}_{K}$, implying that two different $\left\langle M_{\text {ap }}^{2}\right\rangle$ data points are much less correlated compared to the 2PCF. Third, $\left\langle M_{\mathrm{ap}}^{2}\right\rangle$ can be easier extended to higher-order statistics (Schneider et al. 2005). These advantages are valuable 
and should be maintained, but the information content should be improved. Hence, we extend the $\left\langle M_{\mathrm{ap}}^{2}\right\rangle$ data vector by one data point of $\xi_{+}\left(\theta_{0}\right)$, which provides the large-scale information of $\mathcal{P}_{K}$ and call this new data vector $\mathcal{N}$. We perform a likelihood analysis for $\mathcal{N}$, examine its ability to constrain cosmological parameters, and compare it to the two aforementioned data vectors.

This paper is organized as follows. Section 2 summarizes the basic theoretical background of 2PCF and $\left\langle M_{\mathrm{ap}}^{2}\right\rangle$. Next we compare the information content of these two second-order measures and introduce the improvement to the $\left\langle M_{\mathrm{ap}}^{2}\right\rangle$ data vector (Sect. 3). We perform a detailed likelihood analysis for the three data vectors and present the results in Sects. 4 and 5. In Sect. 6 we contaminate our shear data vectors with B-modes and again perform the likelihood analysis to investigate how significantly each data vector is influenced. Finally in Sect. 7, we discuss the results and give our conclusions. One final remark should be made on the notation. Both $\xi$ and $\left\langle M_{\mathrm{ap}}^{2}\right\rangle$ denote theoretical quantities calculated from a given power spectrum, whereas $\hat{\xi}$ and $\mathcal{M}$ are estimators obtained by averaging over many data points inside a bin. Vectors and matrices are written in bold font.

\section{Two-point statistics of cosmic shear}

In this section we briefly review the basics of two-point statistics, definitions of shear estimators and corresponding covariances, closely following the paper of Schneider et al. (2002a). For more details on these topics, the reader is referred to Bartelmann \& Schneider (2001) or, more recently, Schneider (2006).

\subsection{Two-point correlation function and aperture mass dispersion}

To measure the shear signal we define $\boldsymbol{\theta}$ as the connecting vector of two points and specify tangential and cross-component of the shear $\gamma$ as

$\gamma_{\mathrm{t}}=-\operatorname{Re}\left(\gamma \mathrm{e}^{-2 \mathrm{i} \varphi}\right) \quad$ and $\quad \gamma_{\times}=-\operatorname{Im}\left(\gamma \mathrm{e}^{-2 \mathrm{i} \varphi}\right)$,

where $\varphi$ is the polar angle of $\boldsymbol{\theta}$. The 2PCFs depend only on the absolute value of $\boldsymbol{\theta}$ and are defined as

$\xi_{ \pm}(\theta)=\left\langle\gamma_{\mathrm{t}} \gamma_{\mathrm{t}}\right\rangle(\theta) \pm\left\langle\gamma_{\times} \gamma_{\times}\right\rangle(\theta)$.

The observed shear field can be decomposed into a gradient component (called E-mode) and a curl component (B-mode) (Crittenden et al. 2002; Schneider et al. 2002b). B-modes are considered to be a contamination of the pure lensing signal, due to noise or unresolved systematics. The limited validity of the Born approximation (Jain et al. 2000) or redshift source clustering (Schneider et al. 2002b) can also create B-modes, although these effects are small. Intrinsic alignment of source galaxies is another possible source of B-modes. Predictions coming from numerical simulations differ on the impact of these effects (e.g. Heavens et al. 2000; Crittenden et al. 2001; Jing 2002). Nevertheless, when photometric redshift information is available, galaxy pairs with similar redshifts can be ignored in the analysis. This excludes a contamination of the signal by intrinsic galaxy alignment and ensures that a measured correlation of galaxy ellipicities is only due to lensing (King \& Schneider 2003). For the case of a general shear field consisting of $E$ - and B-modes, the convergence is also complex, $\kappa=\kappa_{\mathrm{E}}+\mathrm{i} \kappa_{\mathrm{B}}$, and can be related to the shear (Kaiser \& Squires 1993) by

$\kappa_{\mathrm{E}}(\boldsymbol{\theta})+\mathrm{i} \kappa_{\mathrm{B}}(\boldsymbol{\theta})=\frac{1}{\pi} \int \mathrm{d}^{2} \theta^{\prime} \mathcal{D}^{*}\left(\boldsymbol{\theta}-\boldsymbol{\theta}^{\prime}\right) \gamma\left(\boldsymbol{\theta}^{\prime}\right)$, with

$\mathcal{D}^{*}(\boldsymbol{\theta})=\frac{\theta_{2}^{2}-\theta_{1}^{2}+2 \mathrm{i} \theta_{1} \theta_{2}}{|\boldsymbol{\theta}|^{4}}$.

The power spectra of E-mode and B-mode can be defined (Schneider et al. 2002b) using the Fourier transform of $\kappa$

$\left\langle\hat{\kappa}_{\mathrm{E}}(\boldsymbol{\ell}) \hat{\kappa}_{\mathrm{E}}^{*}\left(\boldsymbol{\ell}^{\prime}\right)\right\rangle=(2 \pi)^{2} \delta^{(2)}\left(\boldsymbol{\ell}-\boldsymbol{\ell}^{\prime}\right) \mathcal{P}_{\mathrm{E}}(\ell)$,

$\left\langle\hat{\kappa}_{\mathrm{B}}(\boldsymbol{\ell}) \hat{\kappa}_{\mathrm{B}}^{*}\left(\boldsymbol{\ell}^{\prime}\right)\right\rangle=(2 \pi)^{2} \delta^{(2)}\left(\boldsymbol{\ell}-\boldsymbol{\ell}^{\prime}\right) \mathcal{P}_{\mathrm{B}}(\ell)$,

$\left\langle\hat{\kappa}_{\mathrm{E}}(\boldsymbol{\ell}) \hat{\kappa}_{\mathrm{B}}^{*}\left(\boldsymbol{\ell}^{\prime}\right)\right\rangle=(2 \pi)^{2} \delta^{(2)}\left(\boldsymbol{\ell}-\boldsymbol{\ell}^{\prime}\right) \mathcal{P}_{\mathrm{EB}}(\ell)$,

with $\delta^{(2)}(\boldsymbol{\ell})$ as the two-dimensional Dirac delta distribution. The cross power spectrum $\mathcal{P}_{\mathrm{EB}}$ is expected to vanish for a statistically parity-invariant shear field. Note that $\mathcal{P}_{\mathrm{E}}$ can be related to the power spectrum of density fluctuations $\mathcal{P}_{\delta}$ via Limber's equation (Kaiser 1992, 1998)

$$
\begin{aligned}
\mathcal{P}_{\mathrm{E}}(\ell)= & \frac{9 H_{0}^{4} \Omega_{\mathrm{m}}^{2}}{4 c^{4}} \int_{0}^{w_{\mathrm{h}}} \frac{\mathrm{d} w}{a^{2}(w)} \mathcal{P}_{\delta}\left(\frac{\ell}{f_{K}(w)}, w\right) \\
& \times\left[\int_{w}^{w_{\mathrm{h}}} \mathrm{d} w^{\prime} p_{w}\left(w^{\prime}\right) \frac{f_{K}\left(w^{\prime}-w\right)}{f_{K}\left(w^{\prime}\right)}\right]^{2},
\end{aligned}
$$

with $\ell$ as the Fourier mode on the sky, and $w$ denotes the comoving coordinate, $w_{\mathrm{h}}$ the comoving coordinate of the horizon, $f_{K}(w)$ the comoving angular diameter distance, and $p_{w}$ the redshift distribution of source galaxies. The $2 \mathrm{PCF}$ depend on both power spectra, $\mathcal{P}_{\mathrm{E}}$ and $\mathcal{P}_{\mathrm{B}}$,

$\xi_{+}(\theta)=\int_{0}^{\infty} \frac{\mathrm{d} \ell \ell}{2 \pi} \mathrm{J}_{0}(\ell \theta)\left[\mathcal{P}_{\mathrm{E}}(\ell)+\mathcal{P}_{\mathrm{B}}(\ell)\right]$,
$\xi_{-}(\theta)=\int_{0}^{\infty} \frac{\mathrm{d} \ell \ell}{2 \pi} \mathrm{J}_{4}(\ell \theta)\left[\mathcal{P}_{\mathrm{E}}(\ell)-\mathcal{P}_{\mathrm{B}}(\ell)\right]$,

with $\mathrm{J}_{n}$ denoting the $n$th order Bessel function.

Another second-order cosmic shear measure, the aperture mass dispersion, was introduced by Schneider et al. (1998) and is also related to the power spectrum. In contrast to the 2PCF, $\left\langle\boldsymbol{M}_{\text {ap }}^{2}\right\rangle$ only depends on the E-mode and $\left\langle M_{\perp}^{2}\right\rangle$ only on the B-mode power spectrum, hence the aperture mass statistics provides a powerful tool to separate E- from B-modes

$$
\begin{aligned}
\left\langle\boldsymbol{M}_{\mathrm{ap}}^{2}\right\rangle(\theta) & =\frac{1}{2 \pi} \int_{0}^{\infty} \mathrm{d} \ell \ell \mathcal{P}_{\mathrm{E}}(\ell) W_{\mathrm{ap}}(\theta \ell), \\
\left\langle M_{\perp}^{2}\right\rangle(\theta) & =\frac{1}{2 \pi} \int_{0}^{\infty} \mathrm{d} \ell \ell \mathcal{P}_{\mathrm{B}}(\ell) W_{\mathrm{ap}}(\theta \ell),
\end{aligned}
$$

with

$W_{\text {ap }}(\theta \ell)=\left(\frac{24 \mathrm{~J}_{4}(\ell \theta)}{(\ell \theta)^{2}}\right)^{2}$.

From (9)-(11) we see that the second-order shear measures are filtered versions of $\mathcal{P}_{\mathrm{E}}$ and $\mathcal{P}_{\mathrm{B}}$. How the different filter functions influence the information content of the corresponding measures will be examined more closely in Sect. 3. In practice the aperture mass dispersion is difficult to measure due to gaps and holes in the data field but can be expressed in terms of $\xi_{+}$and $\xi_{-}$as

$$
\left\langle\boldsymbol{M}_{\mathrm{ap}}^{2}\right\rangle(\theta)=\int_{0}^{2 \theta} \frac{\mathrm{d} \vartheta \vartheta}{2 \theta^{2}}\left[\xi_{+}(\vartheta) T_{+}\left(\frac{\vartheta}{\theta}\right)+\xi_{-}(\vartheta) T_{-}\left(\frac{\vartheta}{\theta}\right)\right] .
$$

The explicit calculation and the filter functions $T_{ \pm}$are given in Schneider et al. (2002b). 


\subsection{Estimators}

Consider a sample of galaxies with angular positions $\boldsymbol{\theta}_{i}$. For each pair of galaxies we define the connecting vector $\boldsymbol{\theta}=\boldsymbol{\theta}_{i}-\boldsymbol{\theta}_{j}$ and determine tangential and cross-components of the ellipticities $\left(\epsilon_{\mathrm{t}}\right.$ and $\epsilon_{\times}$) with respect to this connecting vector. From these ellipticities we estimate the $2 \mathrm{PCF}$ in logarithmic bins of $\vartheta$ with a logarithmic bin width $\Delta \vartheta$ (Schneider et al. 2002a). If the bin width is small enough an unbiased estimator for $\xi_{ \pm}(\vartheta)$ is given by

$\hat{\xi}_{ \pm}(\vartheta)=\frac{1}{N_{\mathrm{p}}(\vartheta)} \sum_{i j}\left(\epsilon_{i \mathrm{t}} \epsilon_{j \mathrm{t}} \pm \epsilon_{i \times} \epsilon_{j \times}\right) \Delta_{\vartheta}\left(\left|\boldsymbol{\theta}_{i}-\boldsymbol{\theta}_{j}\right|\right)$,

with $N_{\mathrm{p}}(\vartheta)=\sum_{i j} \Delta_{\vartheta}\left(\left|\boldsymbol{\theta}_{i}-\boldsymbol{\theta}_{j}\right|\right)$ as the number of galaxy pairs inside a bin, and $\Delta_{\vartheta}\left(\left|\boldsymbol{\theta}_{i}-\boldsymbol{\theta}_{j}\right|\right)$ is 1 if $\left|\boldsymbol{\theta}_{i}-\boldsymbol{\theta}_{j}\right|$ lies inside bin $\vartheta$, 0 otherwise. An unbiased estimator of $\left\langle M_{\mathrm{ap}}^{2}\right\rangle$ can be calculated from $\hat{\xi}_{ \pm}(\vartheta)$ using (14),

$\mathcal{M}\left(\theta_{k}\right)=\sum_{i=1}^{I} \frac{\Delta \vartheta_{i} \vartheta_{i}}{2 \theta_{k}^{2}}\left[\hat{\xi}_{+}\left(\vartheta_{i}\right) T_{+}\left(\frac{\vartheta_{i}}{\theta_{k}}\right)+\hat{\xi}_{-}\left(\vartheta_{i}\right) T_{-}\left(\frac{\vartheta_{i}}{\theta_{k}}\right)\right]$,

where $I$ must be chosen such that the upper limit of the $I$ th bin equals twice the value of $\theta_{k}$.

\subsection{Covariances}

Important for characterizing the amount of information of a shear estimator is the corresponding covariance. For the $2 \mathrm{PCF}$ it is defined as

$\mathrm{C}_{\xi}\left(\vartheta_{i}, \vartheta_{j}\right):=\left\langle\left(\xi_{ \pm}\left(\vartheta_{i}\right)-\hat{\xi}_{ \pm}\left(\vartheta_{i}\right)\right)\left(\xi_{ \pm}\left(\vartheta_{j}\right)-\hat{\xi}_{ \pm}\left(\vartheta_{j}\right)\right)\right\rangle$.

Assuming a Gaussian shear field, the covariance of the 2PCF can be calculated analytically (Schneider et al. 2002a; Joachimi et al. 2007). As one already sees from (17), the 2PCF has four different covariances, denoted as $\mathrm{C}_{++}, \mathrm{C}_{+-}, \mathrm{C}_{-+}, \mathrm{C}_{--}$. Only three of them are independent since $\mathrm{C}_{+-}\left(\vartheta_{i}, \vartheta_{j}\right)=\mathrm{C}_{-+}\left(\vartheta_{j}, \vartheta_{i}\right)$. The covariance $\mathrm{C}_{\mathcal{M}}\left(\theta_{k}, \theta_{l}\right)$ of $\mathcal{M}$ is defined analogously. Using (16) we can express $\mathrm{C}_{\mathcal{M}}$ in terms of $\mathrm{C}_{\xi}$ :

$$
\begin{aligned}
\left.\mathrm{C}_{\mathcal{M}}\left(\theta_{k}, \theta_{l}\right)\right)= & \frac{1}{4} \sum_{i=1}^{I} \sum_{j=1}^{J} \frac{\Delta \vartheta_{i} \Delta \vartheta_{j}}{\theta_{k}^{2} \theta_{l}^{2}} \vartheta_{i} \vartheta_{j} \\
& \times\left[\sum_{m, n=+,-} T_{m}\left(\frac{\vartheta_{i}}{\theta_{k}}\right) T_{n}\left(\frac{\vartheta_{j}}{\theta_{l}}\right) \mathrm{C}_{m n}\left(\vartheta_{i}, \vartheta_{j}\right)\right]
\end{aligned}
$$

Similar to (16) $I(J)$ are chosen such that the upper limit of the Ith $\left(J\right.$ th) bin equals twice of $\theta_{k}\left(\theta_{l}\right)$.

\section{The new data vector $\mathcal{N}$}

Consider two data vectors, namely,

$\boldsymbol{\xi}=\left(\begin{array}{l}\boldsymbol{\xi}_{+} \\ \boldsymbol{\xi}_{-}\end{array}\right) \quad$ with $\quad \boldsymbol{\xi}_{+}=\left(\begin{array}{c}\xi_{+}\left(\vartheta_{1}\right) \\ \vdots \\ \xi_{+}\left(\vartheta_{m}\right)\end{array}\right), \boldsymbol{\xi}_{-}=\left(\begin{array}{c}\xi_{-}\left(\vartheta_{1}\right) \\ \vdots \\ \xi_{-}\left(\vartheta_{m}\right)\end{array}\right)$

for the $2 \mathrm{PCF}$ and

$\left\langle\boldsymbol{M}_{\mathrm{ap}}^{2}\right\rangle=\left(\begin{array}{c}\left\langle\boldsymbol{M}_{\mathrm{ap}}^{2}\right\rangle\left(\theta_{1}\right) \\ \vdots \\ \left\langle\boldsymbol{M}_{\mathrm{ap}}^{2}\right\rangle\left(\theta_{n}\right)\end{array}\right)$ for the aperture mass dispersion. The relation (16) can also be written in terms of data vectors and an $n \times 2 m$ transfer matrix $\boldsymbol{A}$

$$
\left\langle\boldsymbol{M}_{\mathrm{ap}}^{2}\right\rangle=\underbrace{\left(\boldsymbol{A}_{+} \mid \boldsymbol{A}_{-}\right)}_{\mathbf{A}}\left(\begin{array}{c}
\boldsymbol{\xi}_{+} \\
\xi_{-}
\end{array}\right),
$$

with $\boldsymbol{A}_{+}$denoting the part of $\boldsymbol{A}$ referring to $\boldsymbol{\xi}_{+}$and $\boldsymbol{A}_{-}$denotes the corresponding part referring to $\boldsymbol{\xi}_{-}$. Equation (21) implies that the information content of $\left\langle\boldsymbol{M}_{\mathrm{ap}}^{2}\right\rangle$ is less than or equal to $\boldsymbol{\xi}$. The amount of information can only be equal if and only if the rank of $\boldsymbol{A}$ equals the dimension of $\boldsymbol{\xi}$, hence rank $\boldsymbol{A}=2 \mathrm{~m}$. We explicitly prove these statements in the Appendix. For the case of $\boldsymbol{\xi}$ and $\left\langle\boldsymbol{M}_{\text {ap }}^{2}\right\rangle, n \leq m$ holds, which can be seen from (16). Therefore the relation (21) is not invertible and the information content of $\left\langle\boldsymbol{M}_{\mathrm{ap}}^{2}\right\rangle$ is smaller compared to $\boldsymbol{\xi}_{ \pm}$. That $\boldsymbol{\xi}_{ \pm}$contains more information on cosmological parameters can also be explained when looking at the filter functions $\mathrm{J}_{0}, \mathrm{~J}_{4}$, and $W_{\text {ap }}$ relating the corresponding second-order shear measures to the underlying power spectrum. The $2 \mathrm{PCF}$, especially $\xi_{+}$, probes the power spectrum over a broad range of Fourier modes and also collects information on scales larger than the survey size. In contrast, the aperture mass dispersion provides a highly localized probe of $\mathcal{P}_{\mathrm{E}}$ and does not contain this large-scale information. Hence, due to the limited field size of a survey, the information content of $\left\langle\boldsymbol{M}_{\mathrm{ap}}^{2}\right\rangle$ is smaller compared to $\xi_{ \pm}$. These considerations lead to the idea of modifying $\left\langle\boldsymbol{M}_{\mathrm{ap}}^{2}\right\rangle$ by adding one data point of $\xi_{+}\left(\theta_{0}\right)$. We define the new data vector $\mathcal{N}$ as

$\mathcal{N}=\left(\begin{array}{c}\left\langle\boldsymbol{M}_{\mathrm{ap}}^{2}\right\rangle\left(\theta_{1}\right) \\ \vdots \\ \left\langle\boldsymbol{M}_{\mathrm{ap}}^{2}\right\rangle\left(\theta_{n}\right) \\ \xi_{+}\left(\theta_{0}\right)\end{array}\right)$

and the corresponding covariance matrix reads

$\mathbf{C}_{\mathcal{N}}=\left(\begin{array}{ccc|c}\mathrm{C}_{\mathcal{M}_{11}} & \cdots & \mathrm{C}_{\mathcal{M}_{1 n}} & \mathrm{C}\left(\mathcal{M}_{1}, \xi_{+}\right) \\ \vdots & \ddots & \vdots & \vdots \\ \mathrm{C}_{\mathcal{M}_{1 n}} & \cdots & \mathrm{C}_{\mathcal{M}_{n n}} & \mathrm{C}\left(\mathcal{M}_{n}, \xi_{+}\right) \\ \hline \mathrm{C}\left(\xi_{+}, \mathcal{M}_{1}\right) & \cdots & \mathrm{C}\left(\xi_{+}, \mathcal{M}_{n}\right) & \mathrm{C}\left(\xi_{+}, \xi_{+}\right)\end{array}\right)$

The upper left $n \times n$ matrix is exactly $\mathbf{C}_{\mathcal{M}}$ and the entry for $\mathrm{C}\left(\xi_{+}, \xi_{+}\right)$is taken from the corresponding covariance matrix of the correlation function. The cross terms can be calculated using (16) to read

$$
\begin{aligned}
\mathrm{C}\left(\mathcal{M}\left(\theta_{k}\right), \hat{\xi}_{+}\left(\theta_{0}\right)\right)= & \frac{1}{2} \sum_{i=1}^{I} \frac{\Delta \vartheta_{i}}{\theta_{k}^{2}} \vartheta_{i}\left[T_{+}\left(\frac{\vartheta_{i}}{\theta_{k}}\right) \mathrm{C}_{++}\left(\vartheta_{i}, \theta_{0}\right)\right. \\
& \left.+T_{-}\left(\frac{\vartheta_{i}}{\theta_{k}}\right) \mathrm{C}_{-+}\left(\vartheta_{i}, \theta_{0}\right)\right]
\end{aligned}
$$

Similar to $\mathbf{C}_{\mathcal{M}}, \mathbf{C}_{\mathcal{N}}$ is almost diagonal, hence data points of different angular scales are hardly correlated. This property vanishes in case we add more than one data point of the 2PCF. Due to their broad filter function $J_{0}$, two or more $2 \mathrm{PCF}$ data points would be strongly correlated, resulting in high off-diagonal terms in $\mathbf{C}_{\mathcal{N}}$. The main intention, namely to include the information of the power spectrum on large angular scales, is already fulfilled by adding one data point of $\xi_{+}$. For small $\ell$ the sampling of the power spectrum by $\xi_{+}(\theta)$ hardly depends on $\theta$, therefore the gain in information on those scales by adding more than one $2 \mathrm{PCF}$ data point would be rather small. 


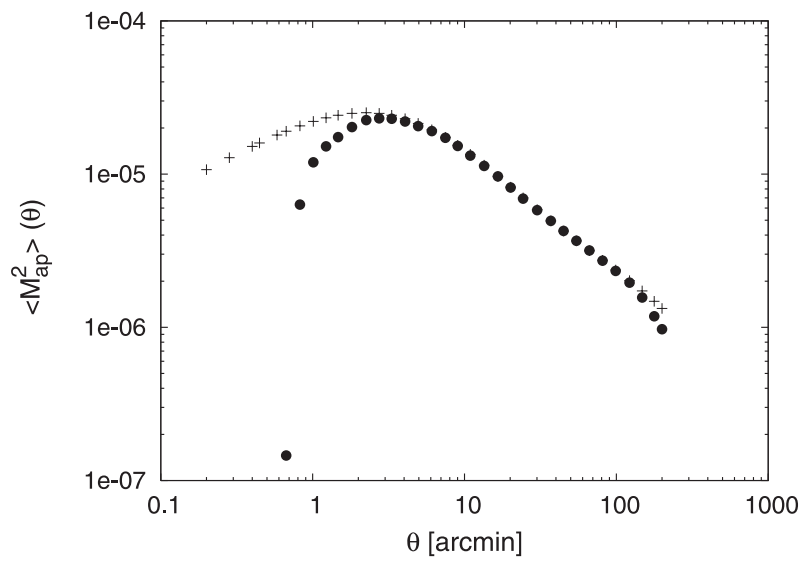

Fig. 1. $\left\langle M_{\text {ap }}^{2}\right\rangle$ calculated directly from the power spectrum (crosses) compared to $\left\langle M_{\mathrm{ap}}^{2}\right\rangle$ calculated from $\xi_{ \pm}$(circles).

\section{Calculating data vectors and covariances}

The data vectors $\boldsymbol{\xi},\left\langle\boldsymbol{M}_{\mathrm{ap}}^{2}\right\rangle, \boldsymbol{N}$ are directly calculated from the power spectrum of density fluctuations $\mathcal{P}_{\delta}$ using (8) to obtain $\mathcal{P}_{\text {E }}$ and then applying either (9), (10), or (11) depending on the desired cosmic shear measure. To derive $\mathcal{P}_{\delta}$ we assume an initial Harrison-Zeldovich power spectrum $\left(\mathcal{P}_{\delta}(k) \propto k^{n}\right.$ with $\left.n=1\right)$. The transition to today's power spectrum employs the transfer function described in Bardeen et al. (1986), and we use the fitting formula of Smith et al. (2003) to calculate the non-linear evolution. In contrast, the covariances are obtained from ray-tracing simulations. The $\mathrm{N}$-body simulation used for the ray-tracing experiment was carried out by the Virgo Consortium (Jenkins et al. 2001); for details of the ray-tracing algorithm see Ménard et al. (2003). Then, $\mathbf{C}_{\xi}$ is calculated by field-to-field variation of 36 ray-tracing realizations, where each field has a sidelength of 4.27 degrees. The intrinsic ellipticity noise is $\sigma_{\epsilon}=0.3$ and the number density of source galaxies is given by $n=25 / \mathrm{arcmin}^{2}$. From $\mathbf{C}_{\xi}$ we calculate $\mathbf{C}_{\mathcal{M}}$ and $\mathbf{C}_{\mathcal{N}}$ according to (18) and (23). Our fiducial cosmological model is determined by the cosmology of the ray-tracing simulations, i.e. a flat $\Lambda \mathrm{CDM}$ model with $\Omega_{\mathrm{m}}=0.3, \sigma_{8}=0.9, h=0.7$, and $\Gamma=0.172$. Furthermore, the ray-tracing simulations assume all source galaxies to be at the same redshift, i.e. $z_{0}=0.98$. Using a redshift distribution instead would not change our results markedly. The data vectors are calculated from $\mathcal{P}_{\mathrm{E}}$, and for a given redshift distribution, one can find a characteristic $z_{0}$ such that $\mathcal{P}_{\mathrm{E}}$ is almost similar independent of using the redshift distribution or choosing all sources to be at $z_{0}$.

\subsection{Difficulties with covariances}

\subsubsection{Underestimation of $\mathbf{C}_{\mathcal{M}}$}

Kilbinger et al. (2006) have shown that $\left\langle M_{\mathrm{ap}}^{2}\right\rangle(\theta)$ is biased for small $\theta$ when calculated from the $2 \mathrm{PCF}$ using (16). This is due to the lack of 2PCF data points on very small angular scales, which causes a small-scale cutoff in the integral of (14). In our specific case the $\left\langle M_{\text {ap }}^{2}\right\rangle$ data vector is not affected by this bias because we calculate it directly from the power spectrum $\mathcal{P}_{\mathrm{E}}$. However, since $\mathbf{C}_{\mathcal{M}}$ and $\mathbf{C}_{\mathcal{N}}$ are calculated from the covariance of the $2 \mathrm{PCF}$, they are certainly affected by this problem. In this subsection we determine the $\theta$-range on which we can calculate $\mathbf{C}_{\mathcal{M}}$ with sufficient accuracy; the corresponding data vector of the aperture mass dispersion will be restricted to this range. Figure 1 shows $\left\langle M_{\text {ap }}^{2}\right\rangle$ calculated directly from the power spectrum using (11) compared with $\left\langle M_{\mathrm{ap}}^{2}\right\rangle$ calculated from $\xi_{ \pm}$using (16). We assume that the deviation shown here is a good approximation of the bias in $\mathbf{C}_{\mathcal{M}}$ and we require an accuracy of $5 \%$ to accept a $\theta$-value for the $\left\langle M_{\mathrm{ap}}^{2}\right\rangle$ data vector. This criterion restricts the data vector to a $\theta$-range of $2.25-100{ }^{\prime} 0$, whereas the $2 \mathrm{PCF}$ data vector is measured from $0.2-200.0$.

\subsubsection{Inversion of the covariance matrix}

A second difficulty in the context of covariance matrices is outlined in Hartlap et al. (2007). The fact that an inversion of an estimated unbiased covariance matrix leads to a biased result can be overcome by applying a correction factor. According to Hartlap et al. (2007), the correction factor depends on the ratio of number of bins $(B)$ to number of independent realizations $(N)$ from which the covariance matrix is estimated. An unbiased estimate of the inverse covariance matrix is

$\mathbf{C}_{\text {unbiased }}^{-1}=\frac{N-B-2}{N-1} \mathbf{C}^{-1}=\left[1-\frac{B+1}{N-1}\right] \mathbf{C}^{-1}$.

Hartlap et al. (2007) prove the validity of this correction factor for the case of Gaussian errors and statistically independent data vectors. These two assumptions are violated when estimating the covariance matrix from ray-tracing simulations. As a check of whether the correction factor corrects the error in our raytracing covariance matrices, we perform the following experiment. We add different Gaussian noise to the ellipticities of the galaxies, which are taken from the 36 independent realizations of the ray-tracing simulations and thereby increase the number of independent realizations. We hold the binning of the matrices constant, calculate covariances for $36,108,216,360,720,1080$, 1440,1800 realizations and plot $1 / \operatorname{tr} \mathbf{C}^{-1}$ depending on the ratio $B / N$ (Fig. 2). Note that this method only creates multiple realizations of Gaussian noise on the galaxy ellipticities and does not increase the number of realizations that determine the cosmic variance part of the covariance matrix. Therefore, this method only partly checks for the non-Gaussianity of the errors in a ray-tracing covariance matrix; nevertheless, the impact of statistically dependent data vectors is fully taken into account. We find the same linear behavior of the bias as Hartlap et al. (2007), so are confident that the correction factor is able to unbias our covariance matrices. By using the corrected inverse covariance matrix we assure that the log-likelihood is also unbiased; nevertheless, any non-linear transformation of the log-likelihood will again introduce a bias that influences the results and must be examined.

\section{Likelihood analysis}

We define the posterior likelihood $\left(P_{\mathrm{PL}}\right)$ for the case of a $2 \mathrm{PCF}$ data vector as

$P_{\mathrm{PL}}(\boldsymbol{\pi} \mid \boldsymbol{\xi})=\frac{P_{\mathrm{L}}(\boldsymbol{\xi} \mid \boldsymbol{\pi})}{P_{\mathrm{E}}(\boldsymbol{\xi})} P_{\text {Prior }}(\boldsymbol{\pi})$,

where $\pi$ denotes the parameter vector of the $\Lambda$ CDM model assumed in our likelihood analysis. Prior knowledge of the parameter vector coming from other experiments is included in $P_{\text {Prior }}$. In our case we assume flat priors with cutoffs, which means $P_{\text {Prior }}$ is constant for all parameters inside a fixed interval and $P_{\text {Prior }}=0$ for parameters outside the interval. The evidence $P_{\mathrm{E}}$, is the normalization, obtained by integrating the probability over the whole parameter space. The likelihood $P_{\mathrm{L}}$ is defined as

$P(\boldsymbol{\xi} \mid \boldsymbol{\pi})=\frac{1}{(2 \pi)^{n / 2} \sqrt{\operatorname{det} \mathbf{C}_{\xi}}} \exp \left[-\frac{1}{2} \chi^{2}(\boldsymbol{\xi}, \boldsymbol{\pi})\right]$, 

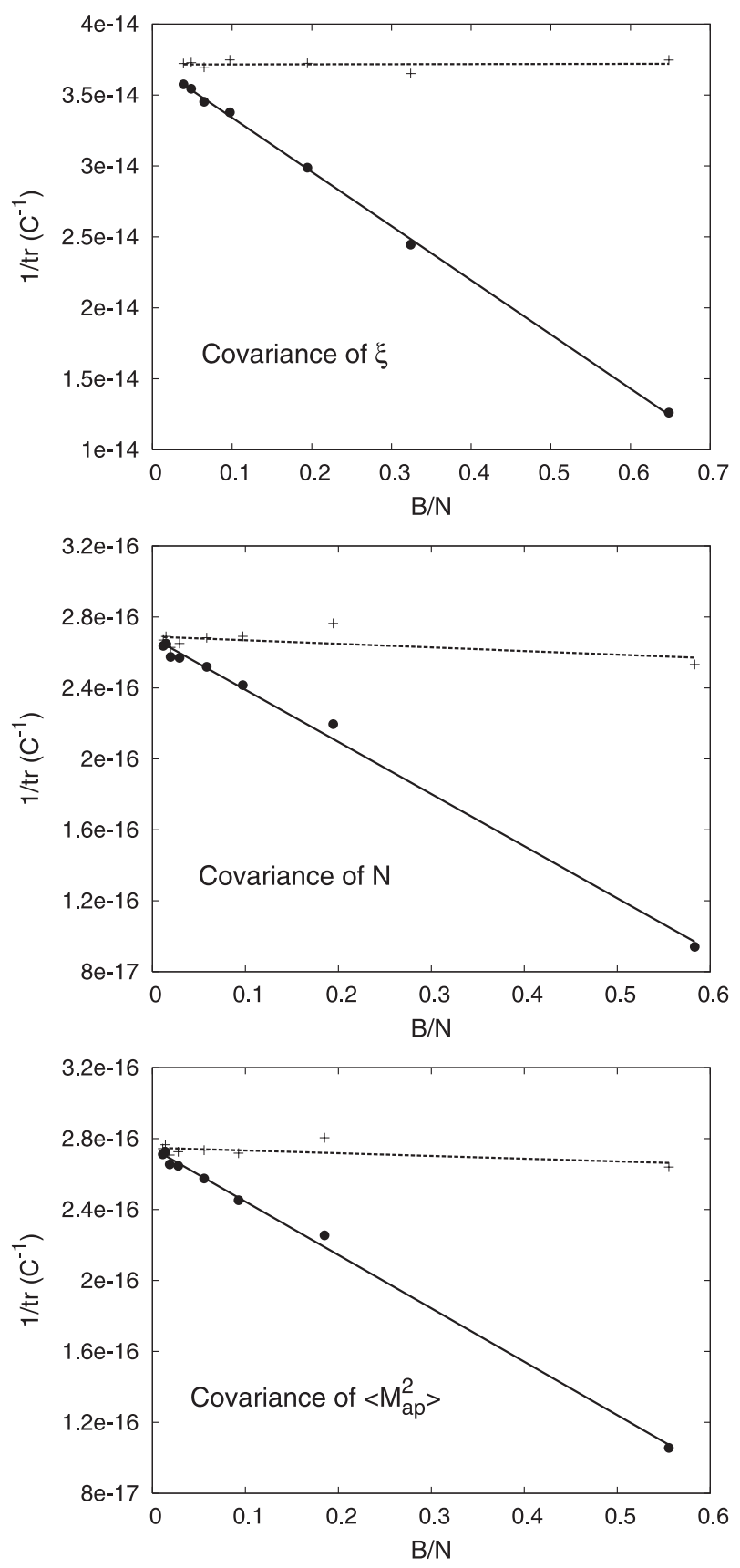

Fig. 2. Illustration of how to correct for the bias occurring in the inverted covariance matrices. We plot $1 /$ trace $\left(\mathbf{C}^{-1}\right)$ against the ratio $B / N$, where $B$ is the number of bins in the covariance matrix and $N$ the number of independent realizations of the ray-tracing simulations. From top to bottom we show the correction for the inverse of $\mathbf{C}_{\xi}, \mathbf{C}_{\mathcal{N}}$, and $\mathbf{C}_{\mathcal{M}}$; each plot shows the corrected (crosses) and uncorrected (circles) values with the corresponding linear fits through the data points.

with the $\chi^{2}$-function

$\chi^{2}(\boldsymbol{\xi}, \boldsymbol{\pi})=\left(\boldsymbol{\xi}(\boldsymbol{\pi})-\xi^{\mathrm{f}}\right)^{\mathrm{t}} \mathbf{C}_{\xi}^{-1}\left(\boldsymbol{\xi}(\boldsymbol{\pi})-\boldsymbol{\xi}^{\mathrm{f}}\right)$,

where $\xi^{\mathrm{f}}$ denotes the data vector corresponding to our fiducial model, whereas $\boldsymbol{\xi}(\boldsymbol{\pi})$ varies according to the considered parameter space. To compare the information content of $\boldsymbol{\xi},\left\langle\boldsymbol{M}_{\mathrm{ap}}^{2}\right\rangle, \mathcal{N}$ we calculate the posterior likelihood in several parameter spaces and illustrate the result by contour plots. Smaller contours correspond to higher information content.

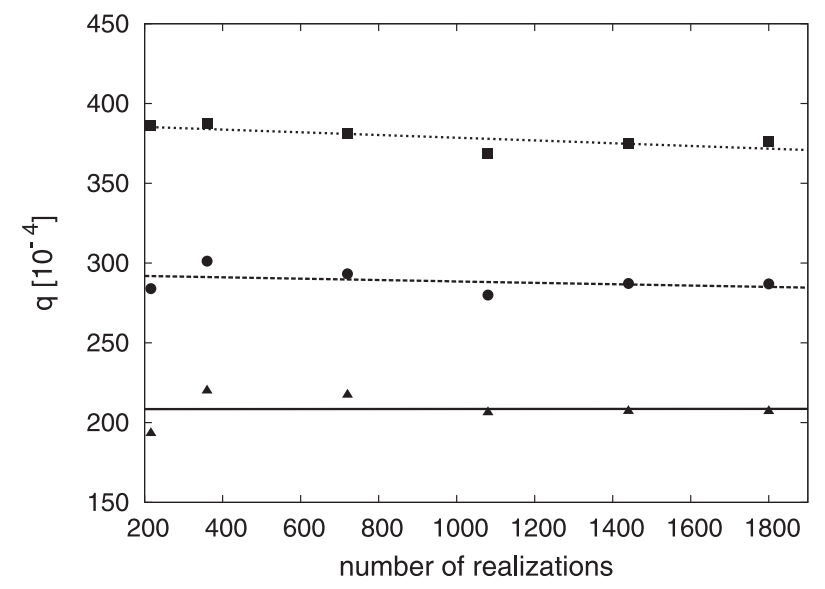

Fig. 3. Dependence of $q$ s calculated for $\boldsymbol{\xi}$ (triangles), $\mathcal{N}$ (circles), $\left\langle\boldsymbol{M}_{\mathrm{ap}}^{2}\right\rangle$ (squares) on the numbers of independent realizations of the ray-tracing simulation. The values of $q$ s are calculated for the case of varying $\Omega_{\mathrm{m}}$ vs. $\sigma_{8}$ in parameter space. The lines are a linear fit through data points (solid for $\boldsymbol{\xi}$, dashed for $\mathcal{N}$, dotted for $\left\langle\boldsymbol{M}_{\text {ap }}^{2}\right\rangle$ ). Note that the deviation of $q$ s belonging to different numbers of realizations is much smaller compared to the difference of $q$ s of different data vectors.

\subsection{Quadrupole moments}

In addition to contour plots, we illustrate the information content of a data vector by calculating the determinant of the quadrupole moment of the posterior likelihood (Kilbinger \& Schneider 2004)

$Q_{i j} \equiv \int \mathrm{d}^{2} \pi P_{\mathrm{PL}}\left(\pi_{1}, \pi_{2}\right)\left(\pi_{i}-\pi_{i}^{\mathrm{f}}\right)\left(\pi_{j}-\pi_{j}^{\mathrm{f}}\right)$

with $\pi_{1}$ and $\pi_{2}$ the varied parameters, and $\pi_{i}^{\mathrm{f}}$ the parameter of the fiducial model. The calculation of $Q_{i j}$ assumes a posterior likelihood in a two-dimensional parameter space; when considering more than two varied parameters, we calculate the $Q_{i j}$ for the marginalized posterior likelihood (see Sect. 5.3). The determinant is given by

$q=\sqrt{\operatorname{det} Q_{i j}}=\sqrt{Q_{11} Q_{22}-Q_{12}^{2}}$

Tighter constraints on the parameters correspond to a lower value of $q$. Due to its non-linearity in the log-likelihood, $q$ is biased (Sect. 4.1.2). The amount of bias varies depending on the number of independent realizations from which the covariance matrix is estimated, and we examine this effect in the same way as for the covariance matrices in Sect. 4.1.2. For six different numbers of independent realizations we performed a likelihood analysis in a two-parameter space $\left(\Omega_{\mathrm{m}}\right.$ vs. $\left.\sigma_{8}\right)$ and calculated $q$ for all three cosmic shear measures. The result is plotted in Fig. 3. One clearly sees that the $q$ dependence on the number of realizations is much weaker compared with the difference between $q$ of different cosmic shear measures. Therefore, the bias is small and we can confidently use $q$ to compare the relative information content of the different data vectors.

\subsection{Variation of two parameters}

The likelihood analysis in this section was performed in a twodimensional parameter space; all other cosmological parameters were fixed to the fiducial values. Before comparing the three data vectors we optimized $\mathcal{N}$ with respect to the $\theta_{0}$-value of the added 2PCF data point. We added 35 different $\xi_{+}\left(\theta_{0}\right)$ covering a range 

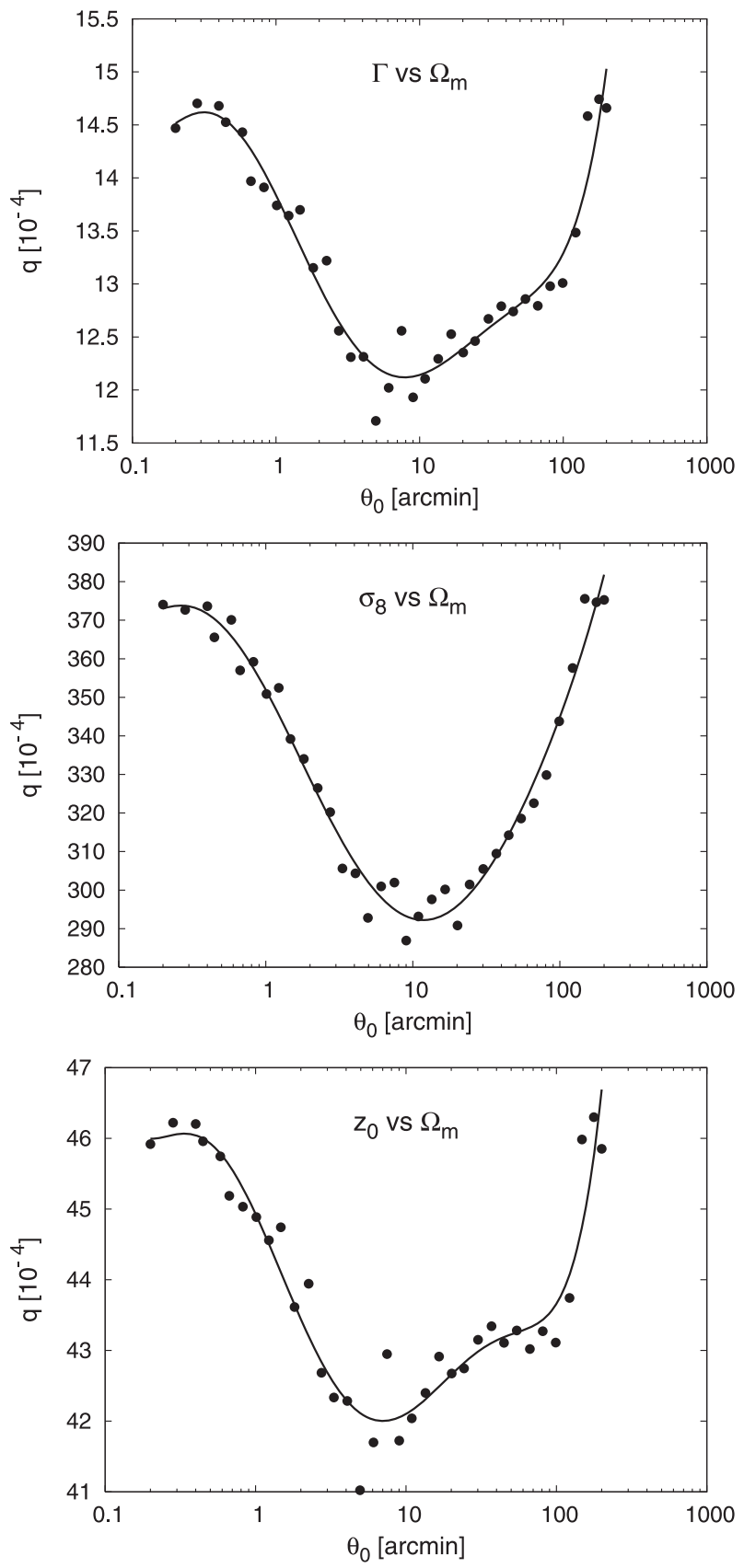

Fig. 4. Values of $q$ of the combined data vector $\mathcal{N}$ depending on the angular separation $\left(\theta_{0}\right)$ of the additional $\xi_{+}$data point. We consider three different parameter spaces $\Gamma$ vs. $\Omega_{\mathrm{m}}$ (top), $\sigma_{8}$ vs. $\Omega_{\mathrm{m}}$ (middle), and $z_{0}$ vs. $\Omega_{\mathrm{m}}$ (bottom), and for each case we calculated $q$ for 35 different added $\xi_{+}\left(\theta_{0}\right)$. The solid lines indicate a polynomial fit through the data; the minima of these fits and therefore the optimal values for $\theta_{0}$ are $7: 8$ $\left(\Gamma\right.$ vs. $\left.\Omega_{\mathrm{m}}\right), 12^{\prime} .9\left(\sigma_{8}\right.$ vs. $\left.\Omega_{\mathrm{m}}\right)$, and 7'.0 ( $z_{0}$ vs. $\left.\Omega_{\mathrm{m}}\right)$.

$\theta_{0} \in\left[0.2-200{ }^{\prime} 0\right]$ and calculated $q$. Figure 4 illustrates the results of this optimization for 3 different pairs of parameters $(\Gamma$ vs. $\Omega_{\mathrm{m}}, \sigma_{8}$ vs. $\Omega_{\mathrm{m}}, z_{0}$ vs. $\left.\Omega_{\mathrm{m}}\right)$. For all parameter combinations considered, the optimal $\theta_{0}$ is close to $10^{\prime}$. This can be explained from the behavior of the covariance matrix. For small angular scales the covariance is dominated by shot noise, whereas the signal of $\xi_{+}$becomes very small for large angular scales. In both cases the signal-to-noise ratio is lower than on medium angular scales, where we find the minimum of $q$. In our later analysis we always chose the optimal $2 \mathrm{PCF}$ data point for the combined data vector. The results are illustrated by contour plots (Fig. 5) and the corresponding values of $q$ are summarized in Table 1 . Here, we also list the results for two additional parameter combinations, $\sigma_{8}$ vs. $\Gamma$ and $z_{0}$ vs. $\sigma_{8}$, not shown in Fig. 5 . One clearly sees that the $2 \mathrm{PCF}$ data vector gives the tightest constraints on cosmological parameters, whereas constraints from the aperture mass dispersion are weaker. Although not quite matching the amount of information of $\boldsymbol{\xi}$, the combined data vector is a substantial improvement over $\left\langle\boldsymbol{M}_{\mathrm{ap}}^{2}\right\rangle$. This result is consistent for all parameter combinations we examine; nevertheless, the amount of the improvement varies. We calculated the difference in information of $\boldsymbol{\xi}$ and $\mathcal{N}$ relative to $\left\langle\boldsymbol{M}_{\mathrm{ap}}^{2}\right\rangle$ and denote these values $\Delta \boldsymbol{\xi}$ and $\Delta \mathcal{N}$ (Table 1). The parameter combination $\sigma_{8}$ vs. $\Omega_{\mathrm{m}}$ shows a relative improvement of $\Delta \mathcal{N}=26.4 \%$, whereas the improvement is much less for the case $z_{0}$ vs. $\sigma_{8}(\Delta \mathbf{N}=4.1 \%)$. The amount of new information contributed by $\xi_{+}\left(\theta_{0}\right)$ depends on two main issues. First, $\xi_{+}$integrates over a very broad range of the power spectrum and it can happen that, although $\mathcal{P}_{\mathrm{E}}$ is sensitive to the parameters considered, the integral over $\mathcal{P}_{K}$ is much less. For example, if one varies $\Gamma$, the power spectrum is tilted and looks significantly different, whereas the corresponding $\xi_{+}\left(\theta_{0}\right)$ might be very similar. Second, $\left\langle\boldsymbol{M}_{\mathrm{ap}}^{2}\right\rangle$ does not contain information on small Fourier modes, whereas $\mathcal{N}$ gains information about these modes from the data point $\xi_{+}\left(\theta_{0}\right)$. However, in case these modes of the power spectrum are not sensitive to the parameters considered, the information that is contributed by $\xi_{+}\left(\theta_{0}\right)$ is mainly redundant, hence $\Delta \mathcal{N}$ is low. For example, varying $\sigma_{8}$ or $\Omega_{\mathrm{m}}$ changes $\mathcal{P}_{\mathrm{E}}$ similarly, i.e. increasing $\Omega_{\mathrm{m}}$ or $\sigma_{8}$ increases the amplitude of $\mathcal{P}_{\mathrm{E}}$ on all Fourier modes. Therefore, the integration over $\mathcal{P}_{\mathrm{E}}$ is as sensitive to parameter variations as $\mathcal{P}_{\mathrm{E}}$ itself. Furthermore, the deviation of power spectra with different values in $\sigma_{8}$ and $\Omega_{\mathrm{m}}$ becomes much more significant for small Fourier modes. Information on these scales is not included in $\left\langle\boldsymbol{M}_{\mathrm{ap}}^{2}\right\rangle$ but is contributed by $\xi_{+}\left(\theta_{0}\right)$, resulting in a large $\Delta \mathbf{N}(26.4 \%)$. In contrast to this, a variation in $z_{0}$ changes the power spectum very little, especially the dependence is weak on low $\ell$-scales. Accordingly, the gain in information for the cases $z_{0}$ vs. $\Omega_{\mathrm{m}}$ and $z_{0}$ vs. $\sigma_{8}$ is rather small.

\subsection{Variation of three and four parameters - marginalization}

In this section we perform a likelihood analysis in three- and four-dimensional parameter space. To illustrate the results in two-dimensional contour plots, we define the marginalized posterior likelihood

$$
P_{\mathrm{mPL}}\left(\boldsymbol{\pi}_{12} \mid \boldsymbol{\xi}_{ \pm}\right)=\int \mathrm{d} \pi_{3} \int \mathrm{d} \pi_{4} P_{\mathrm{PL}}\left(\boldsymbol{\pi}_{1234} \mid \boldsymbol{\xi}_{ \pm}\right)
$$

which is obtained by integrating over the posterior likelihood of the marginalized parameters. The marginalized likelihood is also biased due to its non-linearity in the log-likelihood. To examine whether this bias affects our results significantly, we performed the same experiment as for $q$ in two-dimensional parameter space. We calculated $q$ for our three different measures depending on the number of realizations. The results are shown in Fig. 6; again, the bias due to the process of marginalization is small compared to the difference in $q$ of our three data vectors showing that in the marginalized case we can also use $q$ to compare the information content. We also optimize the combined data vector, similar to Sect. 5.2 and summarize the results in Table 2. For the same reasons as in the previous section, the optimal angular scale of the added $\xi_{+}$data point is again around $10^{\prime}$, and we choose this optimized $\mathcal{N}$ for the likelihood analysis in three- and four-dimensional parameter space. The results of the 

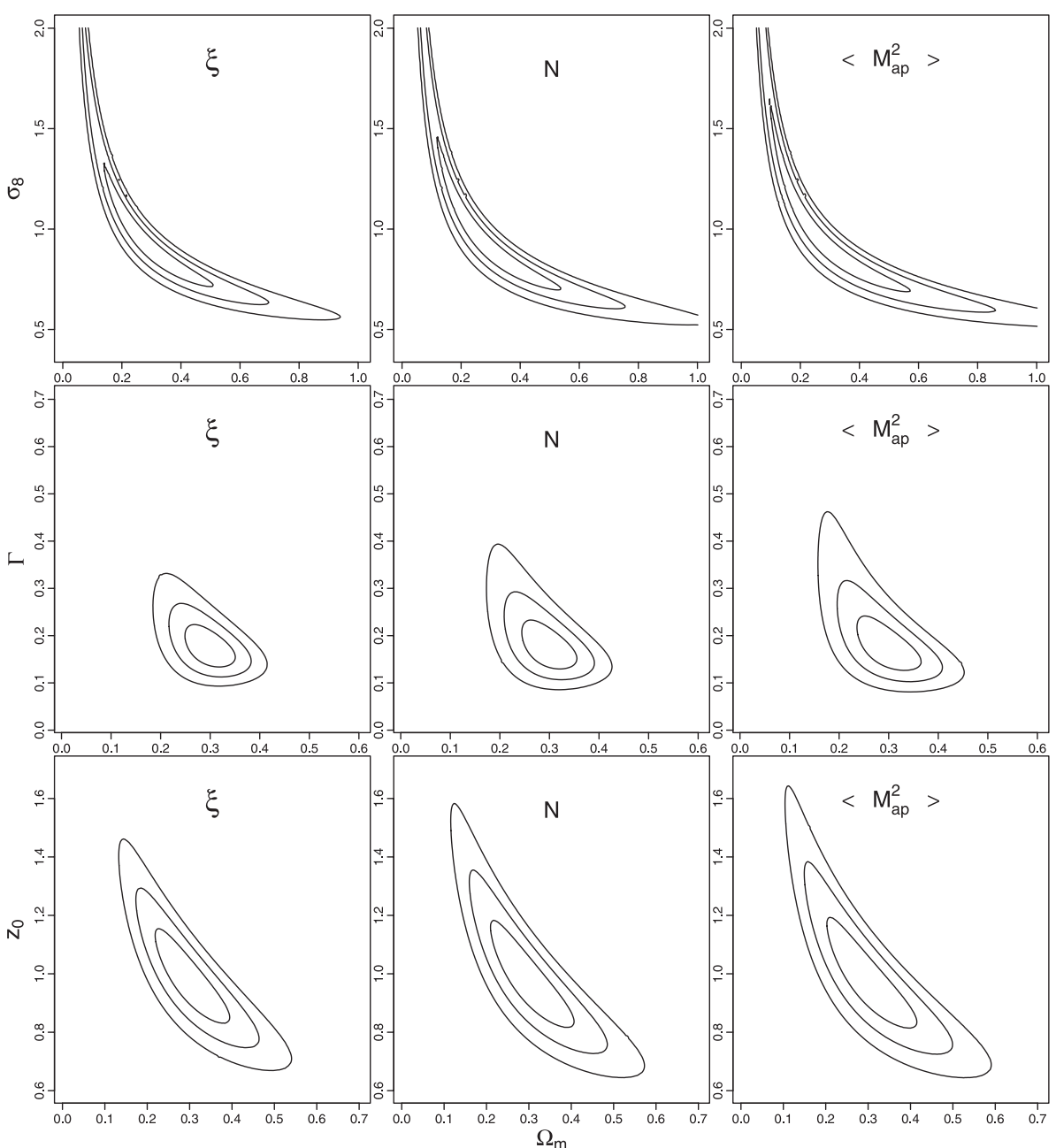

Fig. 5. Likelihood contours when varying only two parameters, while the others are fixed to the fiducial values. The contours contain $68.3 \%$, $95.4 \%, 99.73 \%$ of the posterior likelihood. We consider 3 parameter spaces, from top to bottom: $\sigma_{8}$ vs. $\Omega_{\mathrm{m}}, \Gamma$ vs. $\Omega_{\mathrm{m}}, z_{0}$ vs. $\Omega_{\mathrm{m}}$. The constraints of $\boldsymbol{\xi}$ are shown on the left, $\mathcal{M}$ is plotted in the middle, and the results of $\left\langle\boldsymbol{M}_{\mathrm{ap}}^{2}\right\rangle$ are shown on the right.
Table 1. Values of $q$ for $\boldsymbol{\xi}, \mathcal{N}$, and $\left\langle\boldsymbol{M}_{\text {ap }}^{2}\right\rangle$ considering various parameter spaces.

\begin{tabular}{llllll}
\hline \hline Parameter space & $q\left(\left\langle\boldsymbol{M}_{\mathrm{ap}}^{2}\right\rangle\right)$ & $q(\boldsymbol{N})$ & $q(\boldsymbol{\xi})$ & $\Delta q(\boldsymbol{N})$ & $\Delta q(\boldsymbol{\xi})$ \\
\hline$\Gamma$ vs. $\Omega_{\mathrm{m}}$ & 14.7 & 11.7 & 9.1 & $20.4 \%$ & $38.1 \%$ \\
$\sigma_{8}$ vs. $\Gamma$ & 23.1 & 19.0 & 14.6 & $17.8 \%$ & $36.8 \%$ \\
$\sigma_{8}$ vs. $\Omega_{\mathrm{m}}$ & 376.3 & 286.9 & 207.1 & $23.7 \%$ & $45.0 \%$ \\
$z_{0}$ vs. $\Omega_{\mathrm{m}}$ & 46.4 & 41.0 & 32.9 & $11.6 \%$ & $29.1 \%$ \\
$z_{0}$ vs. $\sigma_{8}$ & 95.3 & 91.4 & 73.2 & $4.1 \%$ & $23.2 \%$ \\
\hline$\sigma_{8}$ vs. $\Omega_{\mathrm{m}}\left(z_{0}\right)$ & 416.9 & 313.4 & 230.0 & $25.8 \%$ & $44.8 \%$ \\
$\sigma_{8}$ vs. $\Omega_{\mathrm{m}}(\Gamma)$ & 780.5 & 720.9 & 527.0 & $7.6 \%$ & $32.5 \%$ \\
$\Gamma$ vs. $\Omega_{\mathrm{m}}\left(\sigma_{8}\right)$ & 93.7 & 77.6 & 61.6 & $17.2 \%$ & $34.3 \%$ \\
\hline$\sigma_{8}$ vs. $\Omega_{\mathrm{m}}\left(\Gamma, z_{0}\right)$ & 983.8 & 850.6 & 623.5 & $13.5 \%$ & $36.6 \%$ \\
\hline
\end{tabular}

Note: Parameters over which we marginalize are mentioned in brackets, the $q$ s are given in units of $10^{-4}$, and $\Delta \mathcal{N}(\Delta \boldsymbol{\xi})$ gives the relative improvement compared to the $q$ of $\left\langle\boldsymbol{M}_{\text {ap }}^{2}\right\rangle$.

likelihood analysis are comparable to those obtained in twodimensional parameter space. The $q$ (see Table 1) are larger and the contours (see Fig. 7) broader. Again, the relative improvement $\Delta \mathbf{N}$ depends on the parameter space considered. For $\sigma_{8}$ vs. $\Omega_{\mathrm{m}}$ marginalized over $z_{0}$, the improvement is very high $(25.8 \%)$ but becomes much lower for $\sigma_{8}$ vs. $\Omega_{\mathrm{m}}$ marginalized over $\Gamma$. This can be explained by looking how $\mathcal{P}_{\mathrm{E}}$ changes with respect to the variation in parameter space. For the combination $\sigma_{8}$ vs. $\Omega_{\mathrm{m}}$, we already explained this in Sect. 5.2 and the influence of $z_{0}$ on $\mathcal{P}_{\mathrm{E}}$ is quite similar. Increasing $z_{0}$ also increases $\mathcal{P}_{\mathrm{E}}$, although the effect is not very large. Therefore, the improvement of $\sigma_{8} \mathrm{vs}$. $\Omega_{\mathrm{m}}$ marginalized over $z_{0}$ is comparable to the non-marginalized case. When varying the shape parameter $\Gamma, \mathcal{P}_{\mathrm{E}}$ is tilted and this dependence of $\mathcal{P}_{\mathrm{E}}$ on $\Gamma$ is different compared to the other three parameters. Scales of $\mathcal{P}_{\mathrm{E}}$ which are most sensitive to $\Gamma$ differ from scales sensitive to $\sigma_{8}, \Omega_{\mathrm{m}}$ and $z_{0}$ and the same argument holds for the scales of the added $\xi_{+}\left(\theta_{0}\right)$. Therefore, the optimal $\theta_{0}$ for the case $\sigma_{8}$ vs. $\Omega_{\mathrm{m}}$ marginalized over $\Gamma$ is a compromise and the relative improvement is much lower (7.6\%) compared to $\sigma_{8}$ vs. $\Omega_{\mathrm{m}}$ marginalized over $z_{0}(25.8 \%)$.

\section{Simulation of a B-mode contamination on small angular scales}

In this section we simulate a B-mode contamination of $\boldsymbol{\xi}, \mathcal{N}$, and $\left\langle\boldsymbol{M}_{\mathrm{ap}}^{2}\right\rangle$ on small angular scales. At present there is no model available to describe B-modes; taking into account that B-modes most likely occur on small angular scales (e.g. Hoekstra et al. 2002; van Waerbeke et al. 2005; Massey et al. 2007), we use the following arbitrary model for a B-mode power spectrum

$\mathcal{P}_{\mathrm{B}}(\ell)=0.2 \mathcal{P}_{\mathrm{E}}(\ell) \mathrm{e}^{-\ell_{\mathrm{B}} / \ell}$,

where $\ell_{\mathrm{B}}$ defines a scale beyond which the B-mode contamination decreases quickly. The B-mode contribution to $\xi$ can be calculated from (9) and (10) by assuming $\mathcal{P}_{\mathrm{E}}=0$. To calculate the covariance $\mathbf{C}_{\mathrm{B}}$, we assume that the probability distribution of B-modes can be described by a Gaussian random field. This assumption enables us to calculate the covariance directly in terms 


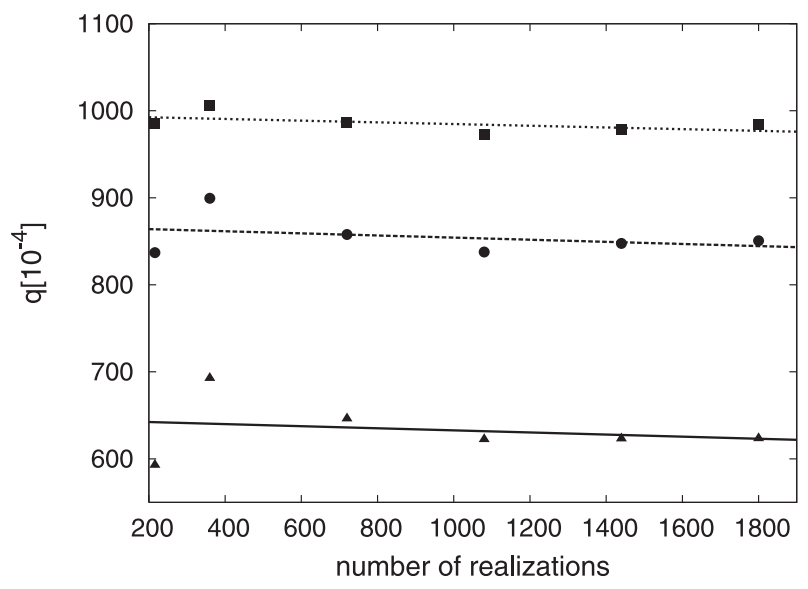

Fig. 6. Dependence of $q$ s calculated for $\boldsymbol{\xi}$ (triangles), $\mathcal{N}$ (circles), and $\left\langle\boldsymbol{M}_{\mathrm{ap}}^{2}\right\rangle$ (squares) on the numbers of realizations for the case of a marginalized posterior likelihood. The parameter space is $\sigma_{8}$ vs. $\Omega_{\mathrm{m}}$ (marginalized over $\Gamma$ and $z_{0}$ ). The lines are a linear fit through data points (solid for $\boldsymbol{\xi}$, dashed for $\mathcal{N}$, dotted for $\left\langle\boldsymbol{M}_{\mathrm{ap}}^{2}\right\rangle$ ). Similar to Fig. 3, the deviation of $q \mathrm{~s}$ belonging to different numbers of realizations is much smaller compared to the deviation of $q$ s of different measures.

Table 2. The optimal angular separation $\theta_{0}$ of the added $\xi_{+}$data point in $\mathcal{N}$ considering three- and four-dimensional parameter space.

\begin{tabular}{ll}
\hline \hline Parameter space & Optimal value $\theta_{0}$ \\
\hline$\Gamma$ vs. $\Omega_{\mathrm{m}}\left(\sigma_{8}\right)$ & $\theta_{0}=9{ }^{\prime} 1$ \\
$\sigma_{8}$ vs. $\Omega_{\mathrm{m}}\left(z_{0}\right)$ & $\theta_{0}=13^{\prime} \cdot 0$ \\
$\sigma_{8}$ vs. $\Omega_{\mathrm{m}}\left(\Gamma\right.$ and $\left.z_{0}\right)$ & $\theta_{0}=12^{\prime} .0$ \\
\hline
\end{tabular}

* We marginalize over the parameters mentioned in parenthesis.

of the power spectrum $\mathcal{P}_{\mathrm{B}}$ (Joachimi et al. 2007). The covariance of the $2 \mathrm{PCF}$ corresponding to the $\mathrm{B}$-mode contribution is given by

$$
\begin{aligned}
& \mathrm{C}_{\mathrm{B}, i j}^{++}=\frac{1}{\mathrm{~A} \pi} \int \mathrm{d} \ell \ell \mathrm{J}_{0}\left(\ell \vartheta_{i}\right) \mathrm{J}_{0}\left(\ell \vartheta_{j}\right)\left(\mathcal{P}_{\mathrm{B}}^{2}(\ell)+\mathcal{P}_{\mathrm{B}}(\ell) \frac{\sigma_{\epsilon}^{2}}{n}\right), \\
& \mathrm{C}_{\mathrm{B}, i j}^{--}=\frac{1}{\mathrm{~A} \pi} \int \mathrm{d} \ell \ell \mathrm{J}_{4}\left(\ell \vartheta_{i}\right) \mathrm{J}_{4}\left(\ell \vartheta_{j}\right)\left(\mathcal{P}_{\mathrm{B}}^{2}(\ell)+\mathcal{P}_{\mathrm{B}}(\ell) \frac{\sigma_{\epsilon}^{2}}{n}\right), \\
& \mathrm{C}_{\mathrm{B}, i j}^{+-}=-\frac{1}{\mathrm{~A} \pi} \int \mathrm{d} \ell \ell \mathrm{J}_{0}\left(\ell \vartheta_{i}\right) \mathrm{J}_{4}\left(\ell \vartheta_{j}\right)\left(\mathcal{P}_{\mathrm{B}}^{2}(\ell)+\mathcal{P}_{\mathrm{B}}(\ell) \frac{\sigma_{\epsilon}^{2}}{n}\right),
\end{aligned}
$$

where $A$ defines the solid angle of the data field, $\sigma_{\epsilon}$ the intrinsic ellipticity noise, and $n$ the number density of the source galaxies. According to the corresponding values of the ray-tracing simulations we choose $\sigma_{\epsilon}=0.3$ and $n=25 / \operatorname{arcmin}^{2}$. Note that $\mathrm{C}_{\mathrm{B}, i j}^{-+}=\mathrm{C}_{\mathrm{B}, j i}^{+-}$. The pure shot noise term of $\mathbf{C}_{\mathrm{B}}^{ \pm \pm}$is contained in $\mathbf{C}_{\mathrm{E}}^{ \pm \pm}$, but in case of $\mathbf{C}_{\mathrm{B}}^{+-}$this term vanishes anyway. We further assume that the contamination is independent of the lensing signal, meaning there is no correlation between E- and B-modes. This assumption does not hold in case the B-mode signal is caused by insufficient PSF correction or other systematics, which we will comment on at the end of this section. For the case that B-modes are created independently from E-modes, we can define a combined E/B-mode covariance matrix as

$\mathbf{C}_{\text {tot }}=\mathbf{C}_{\mathrm{E}}+\mathbf{C}_{\mathrm{B}}$.

Having obtained $\mathbf{C}_{\xi}$ as described above, we therefrom calculate $\mathbf{C}_{\mathcal{M}}$ and $\mathbf{C}_{\mathcal{N}}$ and perform a likelihood analysis similar to Sects. 5.2 and 5.3. We only show the results for the $\Omega_{\mathrm{m}}$ vs. $\sigma_{8}$ plane, which are illustrated in Fig. 8 and Table 3. As expected,
$\left\langle\boldsymbol{M}_{\text {ap }}^{2}\right\rangle$ is not affected by the contamination at all. According to (11), there is no contribution of $\mathcal{P}_{\mathrm{B}}$ to the individual data points of $\left\langle\boldsymbol{M}_{\mathrm{ap}}^{2}\right\rangle$, and the same holds for $\boldsymbol{C}_{\mathcal{M}}$. Therefore, it is no surprise that $q$ and contour plots of $\left\langle\boldsymbol{M}_{\mathrm{ap}}^{2}\right\rangle$ are similar to those in Sect. 5.2. In contrast to this, the $2 \mathrm{PCF}$ data vector is strongly affected by the contamination leading to a $q$ that is $32.8 \%$ higher compared to the case when only E-modes are present. Furthermore, there is a significant deviation between the best-fit parameter set $\left(\sigma_{8}=0.76, \Omega_{\mathrm{m}}=0.39\right)$ and the fiducial cosmological model $\left(\sigma_{8}=0.90\right.$ and $\left.\Omega_{\mathrm{m}}=0.30\right)$. Compared to $\boldsymbol{\xi}$ the combined data vector $\mathcal{N}$ is much less contaminated $(9.6 \%)$, and its best-fit parameter set still matches the fiducial model exactly. Considering the $q$ s, one might argue that $\boldsymbol{\xi}$ still gives tighter constraints on the parameters, but this result is biased in favor of $\boldsymbol{\xi}$ due to considering a parameter space only up to $\Omega_{\mathrm{m}}=1.0$. Both measures have different best-fit parameter sets and the likelihood contours are cut off at the limits of the considered parameter space. In such a case, an extension of the parameter space might change the result of comparing the $q$ s qualitatively. Compared to $\left\langle\boldsymbol{M}_{\mathrm{ap}}^{2}\right\rangle$, the information content of $\mathcal{N}$ is still significantly higher, although the relative improvement decreases to $\Delta \mathcal{N}=16.4 \%$, whereas we obtained $\Delta \mathcal{N}=23.7 \%$ when only E-modes were present (see Sect. 5.2). This decrease, due to the contaminated $\xi_{+}\left(\theta_{0}\right)$ data point in $\mathcal{N}$, is another reason not to include more data points of $\xi_{+}$. In the presence of B-modes, additional 2PCF data points would bias the parameter constraints and weaken the results even more, leading to similar deficits to those obtained from the $2 \mathrm{PCF}$ data vector itself. As already mentioned above, the assumption of B-modes being independent of the E-mode signal does not always hold. In case the contamination affects both, E-mode and B-mode signal, the impact on the parameter constraints of the different measures is hard to quantify. When one measures a B-modes signal, it is a common approach to assume that the E-mode signal is contaminated in a similar way, hence one correspondingly increases its error bars. Although this assumption is sensible, there are possible scenarios where the amount of contamination in E- and Bmode differs and the E-mode contamination cannot be quantified at all. Under the assumption that B-modes trace the scales of the E-mode contamination, it is reasonable to exclude those scales from the likelihood analysis. This can be done using $\left\langle\boldsymbol{M}_{\mathrm{ap}}^{2}\right\rangle$ or $\mathcal{N}$, but $\boldsymbol{\xi}$ cannot avoid the contamination due to its broad filter functions.

\section{Conclusions}

Although the $2 \mathrm{PCF}$ and the aperture mass dispersion are both filtered versions of the power spectrum, the first contains more information on $\mathcal{P}_{\mathrm{E}}$ than the latter. The reason for this is that $\xi$ samples the power spectrum over a much broader range and also collects information on scales that are larger than the size of the survey. The data vector $\left\langle\boldsymbol{M}_{\mathrm{ap}}^{2}\right\rangle$ lacks this large-scale information, but yields highly localized information on $\mathcal{P}_{\mathrm{E}}$. Nevertheless $\left\langle\boldsymbol{M}_{\mathrm{ap}}^{2}\right\rangle$ has other advantages. First, due to its narrow filter function, the data points are much less correlated compared to the $2 \mathrm{PCF}$ data points. This leads to a mainly diagonal covariance matrix, which is numerically stabler during the inversion process in a likelihood analysis. Second, when considering higher-order statistics, $\left\langle M_{\mathrm{ap}}^{3}\right\rangle$ is much easier to handle than the three-point correlation function (Schneider et al. 2005), and third, the aperture mass dispersion is only sensitive to E-modes. Based on these considerations we create the combined data vector $\mathcal{N}$, which preserves the advantages of $\left\langle\boldsymbol{M}_{\text {ap }}^{2}\right\rangle$ and additionally provides large-scale information on $\mathcal{P}_{\mathrm{E}}$. This data vector can be 

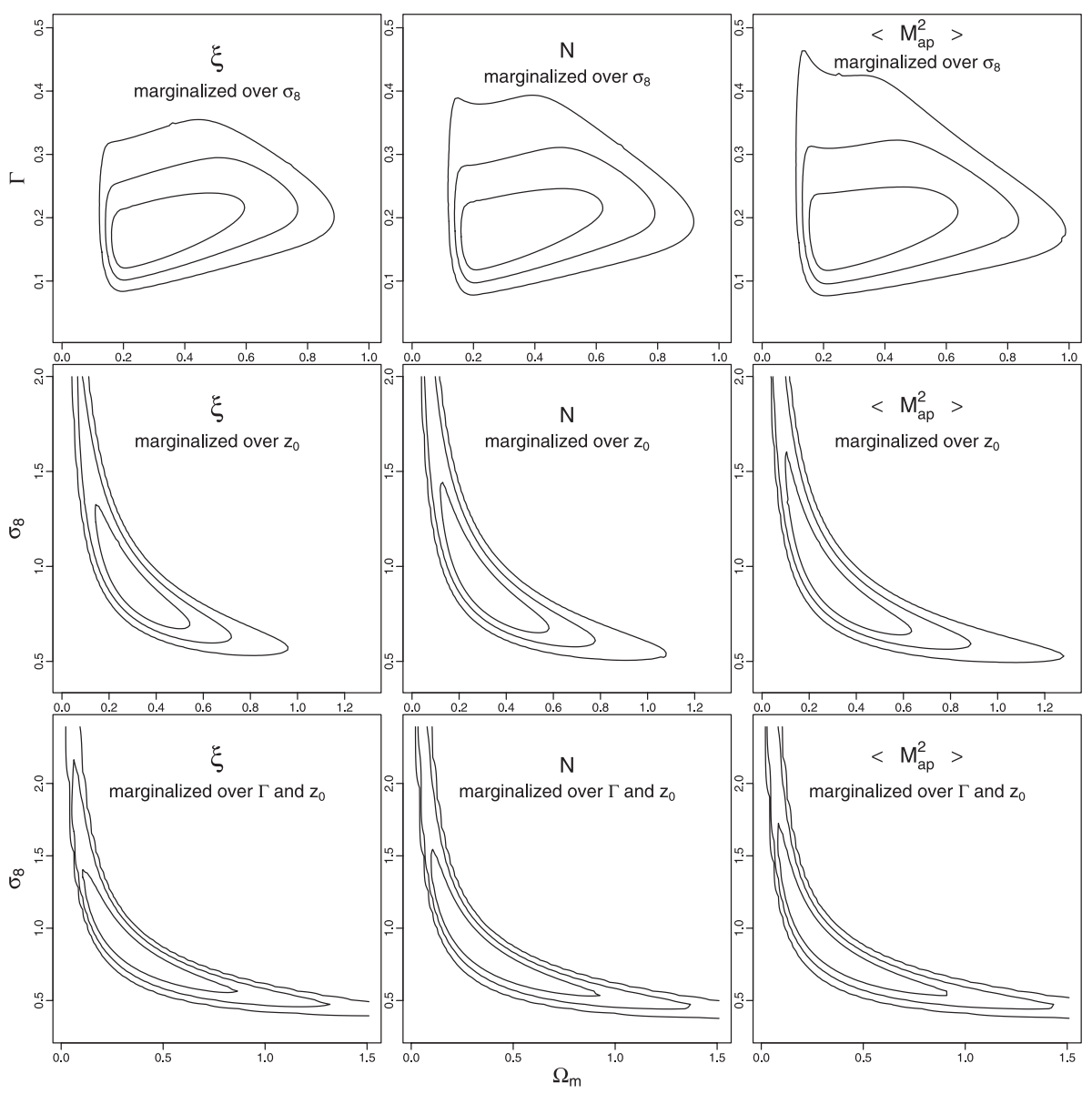

Fig. 7. Likelihood contours of $\boldsymbol{\xi}, \mathcal{N}$, and $\left\langle\boldsymbol{M}_{\mathrm{ap}}^{2}\right\rangle$ in three- and four-dimensional parameter space. From top to bottom we see $\Gamma$ vs. $\Omega_{\mathrm{m}}$ marginalized over $\sigma_{8}, \sigma_{8}$ vs. $\Omega_{\mathrm{m}}$ marginalized over $z_{0}$ and $\sigma_{8}$ vs. $\Omega_{\mathrm{m}}$ marginalized over $\Gamma$ and $z_{0}$. The contours contain $68.3 \%, 95.4 \%$, $99.73 \%$ of the marginalized posterior likelihood. The small scatter of the contours in the last plot is due to a lower resolution of the grid in four-dimensional parameter space compared to the grids in two- and three-dimensional parameter space.

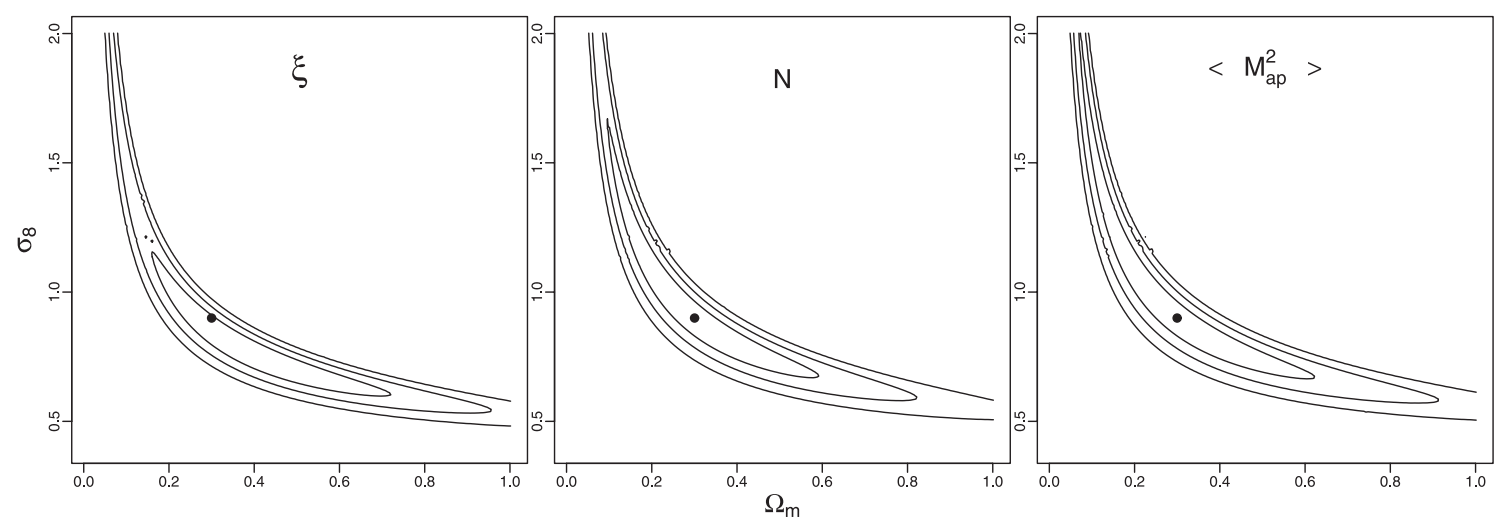

Fig. 8. The likelihood contours for the case that the shear signal is contaminated with B-modes. We only consider a two-dimensional parameter space $\left(\sigma_{8}\right.$ vs. $\left.\Omega_{\mathrm{m}}\right)$ and the contours again contain $68.3 \%, 95.4 \%, 99.73 \%$ of the posterior likelihood. The black dot in each plot indicates the fiducial model.

Table 3. The results of the likelihood analysis in case the data vectors are contaminated by B-modes.

\begin{tabular}{lllll}
\hline \hline Data vector & $q$ (with B-modes) & $q$ (without B-modes) & $\Delta q^{*}$ & Best-fit parameter set \\
\hline$\left\langle\boldsymbol{M}_{\text {ap }}^{2}\right\rangle$ & 376.3 & 376.3 & 0.0 & $\sigma_{8}=0.90, \Omega_{\mathrm{m}}=0.30$ \\
$\mathcal{N}$ & 314.4 & 286.9 & $9.6 \%$ & $\sigma_{8}=0.90, \Omega_{\mathrm{m}}=0.30$ \\
$\boldsymbol{\xi}$ & 275.0 & 207.1 & $32.8 \%$ & $\sigma_{8}=0.76, \Omega_{\mathrm{m}}=0.39$ \\
\hline
\end{tabular}

* The relative difference of the $q$ s with and without B-mode contamination.

optimized with respect to the angular scale of the added data point $\xi_{+}\left(\theta_{0}\right)$, but this optimization very likely depends on the survey geometry and must be performed for each survey separately. We compared the three data vectors in a detailed likelihood analysis and find that the combined data vector is a strong improvement over $\left\langle\boldsymbol{M}_{\mathrm{ap}}^{2}\right\rangle$ in information content. However, the amout of improvement depends on the parameter space considered, more precisely, on the dependence of $\mathcal{P}_{\mathrm{E}}$ on variations in those parameters. The combined data vector $\mathcal{N}$ also maintains the other advantages of the aperture mass dispersion. Its 
covariance matrix is almost diagonal, and even the cross terms $\mathrm{C}\left(\mathcal{M}\left(\theta_{k}\right), \hat{\xi}_{+}\left(\theta_{0}\right)\right)$ are much smaller compared with the offdiagonal terms of $\mathbf{C}_{\xi}$. Comparing the information content of $\boldsymbol{\xi}$ and $\mathcal{N}, \boldsymbol{\xi}$ gives tighter constraints if the shear signal only consists of E-modes. In the more realistic case, when B-modes are also present, the parameter constraints of $\boldsymbol{\xi}$ are significantly weakened and, even worse, biased. The data vector $\mathcal{N}$ is much less affected by the contamination and still gives tighter constraints on cosmological parameters than does $\left\langle\boldsymbol{M}_{\mathrm{ap}}^{2}\right\rangle$.

Acknowledgements. We thank Yannick Mellier, Jan Hartlap, and Tim Schrabback for useful discussions and advice. This work was supported by the Deutsche Forschungsgemeinschaft under the projects SCHN 342/6-1 and SCHN 342/9-1. T.E. is supported by the International Max-Planck Research School at the University of Bonn. M.K. is supported by the CNRS ANR "ECOSSTAT", contract number ANR-05-BLAN-0283-04.

\section{Appendix A: Comparison of two measures}

We compare the information content of two arbitrary data vectors referring to them as primary data vector $\boldsymbol{p}$ and secondary data vector $\boldsymbol{s}$. We further assume that $\boldsymbol{s}$ can be calculated from $\boldsymbol{p}$ by a transfer matrix $\boldsymbol{A}$ (dimension $n \times m$ ), with arbitrary $n$ and $m$

$\boldsymbol{p}=\left(\begin{array}{c}p_{1} \\ p_{2} \\ \vdots \\ p_{m}\end{array}\right) \quad$ and $\quad \boldsymbol{s}=\left(\begin{array}{c}s_{1} \\ s_{2} \\ \vdots \\ s_{n}\end{array}\right) \quad$ with $\quad \boldsymbol{s}=\mathbf{A} \boldsymbol{p}$.

We define the covariance matrices of these data vectors as

$\mathbf{C}_{\mathrm{p}}=\left\langle(\boldsymbol{p}-\hat{\boldsymbol{p}})(\boldsymbol{p}-\hat{\boldsymbol{p}})^{\mathrm{t}}\right\rangle$,

$\mathbf{C}_{\mathrm{s}}=\left\langle(\boldsymbol{s}-\hat{\boldsymbol{s}})(\boldsymbol{s}-\hat{\boldsymbol{s}})^{\mathrm{t}}\right\rangle$,

where $\hat{\boldsymbol{p}}(\hat{\boldsymbol{s}})$ denotes the estimated and $\boldsymbol{p}(\boldsymbol{s})$ the true values of primary (secondary) measure. Using (A.1) we can relate both covariances through

$\mathbf{C}_{\mathrm{s}}=\mathbf{A} \mathbf{C}_{\mathrm{p}} \mathbf{A}^{\mathrm{t}}$

The transformation matrix $\mathbf{A}$ has to be of rank $\mathbf{A}=n$, otherwise the covariance matrix of the secondary data vector $\mathbf{C}_{\mathrm{s}}=$ (A $\mathbf{C}_{\mathrm{p}} \mathbf{A}^{\mathrm{t}}$ ) is singular and not invertible. Furthermore, as $\mathbf{A}$ is of dimension $(n \times m)$, rank $\mathbf{A} \leq m$ implying $n \leq m$. We take the $\chi^{2}$-functions a measure for the information content

$\chi_{\mathrm{p}}^{2}=\Delta_{\mathrm{p}}^{\mathrm{t}} \mathbf{C}_{\mathrm{p}}^{-1} \boldsymbol{\Delta}_{\mathrm{p}} \quad$ and $\quad \chi_{\mathrm{s}}^{2}=\boldsymbol{\Delta}_{\mathrm{s}}^{\mathrm{t}} \mathbf{C}_{\mathrm{s}}^{-1} \boldsymbol{\Delta}_{\mathrm{s}}$,

where in our case $\Delta_{\mathrm{p}}=p^{\mathrm{f}}-\boldsymbol{p}_{\pi}\left(\Delta_{\mathrm{s}}=\boldsymbol{s}^{\mathrm{f}}-\boldsymbol{s}_{\pi}\right)$ denotes the difference between the fiducial data vector $p^{\mathrm{f}}\left(\boldsymbol{s}^{\mathrm{f}}\right)$ and the data vector $\boldsymbol{p}_{\boldsymbol{\pi}}\left(\boldsymbol{s}_{\boldsymbol{\pi}}\right)$ depending on the parameter vector $\boldsymbol{\pi}$. If $\chi^{2}$ is minimal, the posterior likelihood of the corresponding $\pi$ being the correct parameter vector is maximized. The difference between $\chi_{\mathrm{p}}^{2}$ and $\chi_{\mathrm{s}}^{2}$ characterizes which probability function has a larger curvature, i.e. which data vector gives tighter constraints in parameter space. Therefore the information content of primary and secondary data vectors can be compared by calculating

$\chi_{\mathrm{p}}^{2}-\chi_{\mathrm{s}}^{2}=\Delta_{\mathrm{p}}^{\mathrm{t}} \mathbf{C}_{\mathrm{p}}^{-1} \boldsymbol{\Delta}_{\mathrm{p}}-\boldsymbol{\Delta}_{\mathrm{p}}^{\mathrm{t}} \mathbf{A}^{\mathrm{t}}\left(\mathbf{A} \mathbf{C}_{\mathrm{p}} \mathbf{A}^{\mathrm{t}}\right)^{-1} \mathbf{A} \boldsymbol{\Delta}_{\mathrm{p}}$,

for arbitrary $\Delta_{\mathrm{p}}$. In case this difference is always positive, we can conclude that the primary data vector gives tighter constraints on parameters. We can always find transformation matrices $\mathbf{V}$ (dimension $m \times m$ ) and $\mathbf{U}$ (dimension $n \times n)$ to rewrite the transfer matrix $\mathbf{A}$ as an $n \times m$ matrix

$$
\left(\mathbf{E}_{n} \mid \mathbf{0}\right)=\mathbf{S}=\mathbf{U} \mathbf{A} \mathbf{V}^{-1} \longleftrightarrow A=\mathbf{U}^{-1} \mathbf{S} \mathbf{V}
$$

We can directly calculate these transformation matrices as a multiplication of elementary matrices (Fischer 1997a). Inserting (A.7) into (A.6), and after some lengthy but straightforward calculation we derive,

$\chi_{\mathrm{p}}^{2}-\chi_{\mathrm{s}}^{2}=\Delta_{\mathrm{p}}^{\prime \mathrm{t}} \mathbf{C}^{\prime-1} \boldsymbol{\Delta}_{\mathrm{p}}^{\prime}-\boldsymbol{\Delta}_{\mathrm{p}}^{\prime \mathrm{t}} \mathbf{S}^{\mathrm{t}}\left(\mathbf{S} \mathbf{C}^{\prime} \mathbf{S}^{\mathrm{t}}\right)^{-1} \mathbf{S} \boldsymbol{\Delta}_{\mathrm{p}}^{\prime}$

with

$\mathbf{C}^{\prime}=\mathbf{V} \mathbf{C}_{\mathrm{p}} \mathbf{V}^{\mathrm{t}} \quad$ and $\quad \boldsymbol{\Delta}_{\mathrm{p}}^{\prime}=\mathbf{V} \boldsymbol{\Delta}_{\mathrm{p}}$.

For simpler notation we discard all " "” later on. We define

$\mathbf{C}^{-1}=\left(\begin{array}{c|c}\mathbf{C}_{1} & \mathbf{C}_{2} \\ \hline \mathbf{C}_{2}^{\mathrm{t}} & \mathbf{C}_{3}\end{array}\right)^{-1}=\left(\begin{array}{l|l}\mathbf{D}_{1} & \mathbf{D}_{2} \\ \hline \mathbf{D}_{2}^{\mathrm{t}} & \mathbf{D}_{3}\end{array}\right)$,

with $\mathbf{C}_{1}$ being an $n \times n$ matrix and calculate

$\mathbf{S}^{\mathrm{t}}\left(\mathbf{S} \mathbf{C ~ S} \mathbf{S}^{\mathrm{t}}\right)^{-1} \mathbf{S}=\left(\begin{array}{c|c}\mathbf{C}_{1}^{-1} & \mathbf{0} \\ \hline \mathbf{0} & \mathbf{0}\end{array}\right)$

Using (A.10) and (A.11) we can rewrite (A.8) as

$\chi_{\mathrm{p}}^{2}-\chi_{\mathrm{s}}^{2}=\Delta_{\mathrm{p}}^{\mathrm{t}}\left(\begin{array}{c|c}\mathbf{D}_{1}-\mathbf{C}_{1}^{-1} & \mathbf{D}_{2} \\ \hline \mathbf{D}_{2}^{\mathrm{t}} & \mathbf{D}_{3}\end{array}\right) \boldsymbol{\Delta}_{\mathrm{p}}$.

From $\mathbf{C D}=\mathbf{E}_{m}$ we deduce

$\mathbf{C}_{1} \mathbf{D}_{1}+\mathbf{C}_{2} \mathbf{D}_{2}^{\mathrm{t}}=\mathbf{E}_{n} \longrightarrow \mathbf{D}_{1}-\mathbf{C}_{1}^{-1}=-\mathbf{C}_{1}^{-1} \mathbf{C}_{2} \mathbf{D}_{2}^{\mathrm{t}}$

and

$\mathbf{C}_{1} \mathbf{D}_{2}+\mathbf{C}_{2} \mathbf{D}_{3}=0 \longrightarrow \mathbf{C}_{2}=-\mathbf{C}_{1} \mathbf{D}_{2} \mathbf{D}_{3}^{-1}$.

Inserting (A.14) into (A.13) we can rewrite (A.12) as

$\chi_{\mathrm{p}}^{2}-\chi_{\mathrm{s}}^{2}=\Delta_{\mathrm{p}}^{\mathrm{t}}\left(\begin{array}{c|c}\mathbf{D}_{2} \mathbf{D}_{3}^{-1} \mathbf{D}_{2}^{\mathrm{t}} & \mathbf{D}_{2} \\ \hline \mathbf{D}_{2}^{\mathrm{t}} & \mathbf{D}_{3}\end{array}\right) \boldsymbol{\Delta}_{\mathrm{p}}$.

The matrix $\mathbf{C}$ is positive definite and symmetric, therefore $\mathbf{D}_{3}$ as a submatrix is positive definite and symmetric, and the inverse $\mathbf{D}_{3}^{-1}$ also has these favorable properties (Anderson 2003). Hence, we can decompose $\mathbf{D}_{3}=\mathbf{L} \mathbf{L}^{\mathrm{t}}$ and finish our calculation as follows

$$
\begin{aligned}
\chi_{\mathrm{p}}^{2}-\chi_{\mathrm{s}}^{2} & =\Delta_{\mathrm{p}}^{\mathrm{t}}\left(\begin{array}{c}
\mathbf{D}_{2}\left(\mathbf{L}^{\mathrm{t}}\right)^{-1} \\
\mathbf{L}
\end{array}\right) \underbrace{\left(\mathbf{L}^{-1} \mathbf{D}_{2}^{\mathrm{t}} \mathbf{L}\right)}_{\mathbf{T}} \boldsymbol{\Delta}_{\mathrm{p}} \\
& =\boldsymbol{\Delta}_{\mathrm{p}}^{\mathrm{t}} \mathbf{T}^{\mathrm{t}} \mathbf{T} \boldsymbol{\Delta}_{\mathrm{p}} \\
& =\left\|\mathbf{T} \boldsymbol{\Delta}_{\mathrm{p}}\right\|^{2} \\
& \geq 0
\end{aligned}
$$

We now examine the case where $\chi_{\mathrm{p}}^{2}-\chi_{\mathrm{s}}^{2}=0$. The information content of primary and secondary measures is considered to be equal if and only if this equality holds for all data vectors $\Delta_{\mathrm{p}}$. If 
there is only one $\Delta_{\mathrm{p}}$ for which $\chi_{\mathrm{p}}^{2}-\chi_{\mathrm{s}}^{2}>0$, the primary measure contains more information. The difference between the two $\chi^{2}$-values is given by (A.6). In case it is zero for all $\Delta_{p}$,

$$
\mathbf{C}_{\mathrm{p}}^{-1}=\mathbf{A}^{\mathrm{t}}\left(\mathbf{A} \mathbf{C}_{\mathrm{p}} \mathbf{A}^{\mathrm{t}}\right)^{-1} \mathbf{A}
$$

must hold (Fischer 1997b). The matrix $\mathbf{C}_{\mathrm{p}}$ is of rank $m$, hence the lefthandside of (A.18) must also have rank $m$. Then $\mathbf{A}$ must have rank $m$ and is therefore a quadratic $m \times m$ matrix, which is of course invertible. This result is intuitively clear, if one is able to calculate $\Delta_{\mathrm{s}}$ from $\Delta_{\mathrm{p}}$, and vice versa the information content should be the same. We can summarize the results of the above calculation in two statements:

1. If a secondary measure can be calculated from a primary by a matrix $\boldsymbol{A}$ as described in (A.1), the secondary measure has less or equal information.

2. The amount of information is equal in case the rank of $\boldsymbol{A}$ equals the dimension of the primary data vector $(m)$ implying that $\boldsymbol{A}$ is invertible.

\section{References}

Anderson, T. W. 2003, An Introduction to Multivariate Statistical Analysis (Wiley-Interscience), 623/624

Bacon, D., Refregier, A., \& Ellis, R. 2000, MNRAS, 318, 625

Bardeen, J. M., Bond, J. R., Kaiser, N., \& Szalay, A. S. 1986, ApJ, 304, 15

Bartelmann, M., \& Schneider, P. 2001, Phys. Rep., 340, 291

Crittenden, R. G., Natarajan, P., Pen, U.-L., \& Theuns, T. 2001, ApJ, 559, 552
Crittenden, R. G., Natarajan, P., Pen, U.-L., \& Theuns, T. 2002, ApJ, 568, 20

Fischer, G. 1997a, Lineare Algebra (Vieweg), 161

Fischer, G. 1997b, Lineare Algebra (Vieweg), 276

Hartlap, J., Simon, P., \& Schneider, P. 2007, A\&A, 464, 399

Heavens, A., Refregier, A., \& Heymans, C. 2000, MNRAS, 319, 649

Hetterscheidt, M., Simon, P., Schirmer, M., et al. 2007, A\&A, 468, 859

Hoekstra, H., Yee, H. K. C., \& Gladders, M. D. 2002, ApJ, 577, 595

Hoekstra, H., Mellier, Y., van Waerbeke, L., et al. 2006, ApJ, 647, 116

Jain, B., Seljak, U., \& White, S. 2000, ApJ, 530, 547

Jenkins, A., Frenk, C. S., White, S. D. M., et al. 2001, MNRAS, 321, 372

Jing, Y. P. 2002, MNRAS, 335, L89

Joachimi, B., Schneider, P., \& Eifler, T. 2007 [arXiv: 0708.0387v1]

Kaiser, N. 1992, ApJ, 388, 272

Kaiser, N. 1998, ApJ, 498, 26

Kaiser, N., \& Squires, G. 1993, ApJ, 404, 441

Kaiser, N., Wilson, G., \& Luppino, G. A. 2000, ArXiv Astrophysics e-prints

Kilbinger, M., \& Schneider, P. 2004, A\&A, 413, 465

Kilbinger, M., Schneider, P., \& Eifler, T. 2006, A\&A, 457, 15

King, L. J., \& Schneider, P. 2003, A\&A, 398, 23

Massey, R., Rhodes, J., Leauthaud, A., et al. 2007, ArXiv Astrophysics e-prints Ménard, B., Hamana, T., Bartelmann, M., \& Yoshida, N. 2003, A\&A, 403, 817 Schneider, P. 2006, Weak Gravitational Lensing (Berlin: Springer Verlag)

Schneider, P., van Waerbeke, L., Jain, B., \& Kruse, G. 1998, MNRAS, 296, 873

Schneider, P., van Waerbeke, L., Kilbinger, M., \& Mellier, Y. 2002a, A\&A, 396, 1

Schneider, P., van Waerbeke, L., \& Mellier, Y. 2002b, A\&A, 389, 729

Schneider, P., Kilbinger, M., \& Lombardi, M. 2005, A\&A, 431, 9

Schrabback, T., Erben, T., Simon, P., et al. 2007, A\&A, 468, 823

Semboloni, E., Mellier, Y., van Waerbeke, L., et al. 2006, A\&A, 452, 51

Smith, R. E., Peacock, J. A., Jenkins, A., et al. 2003, MNRAS, 341, 1311

van Waerbeke, L., Mellier, Y., Erben, T., et al. 2000, A\&A, 358, 30

van Waerbeke, L., Mellier, Y., \& Hoekstra, H. 2005, A\&A, 429, 75

Wittman, D. M., Tyson, J. A., Kirkman, D., Dell'Antonio, I., \& Bernstein, G.

2000, Nature, 405, 143 